\title{
Wavelet transform for functions with values in UMD spaces
}

\author{
by \\ Cornelia Kaiser (Paderborn) and Lutz Weis (Karlsruhe)
}

\begin{abstract}
We extend the classical theory of the continuous and discrete wavelet transform to functions with values in UMD spaces. As a by-product we obtain equivalent norms on Bochner spaces in terms of g-functions.
\end{abstract}

1. Introduction. In this paper we study the continuous and discrete wavelet transform for functions with values in Banach spaces that have a certain geometrical property (the UMD property). A Banach space $X$ is called a UMD space if the Hilbert transform extends to a bounded operator on $L_{p}(\mathbb{R}, X)$ for some (all) $p \in(1, \infty)$. Most of the reflexive Banach spaces of distributions commonly used in the theory of partial differential equations are UMD spaces (cf. [2, pp. 141-147]).

What we basically will show is that the classical scalar-valued results carry over to the UMD setting. In this sense our results can be considered as an extension and continuation of the work of Figiel. In his paper [13] he proved that certain orthonormal wavelet bases $\left\{\psi_{j, k}\right\}$ are unconditional in $L_{p}\left(\mathbb{R}^{N}, X\right)$, where $p \in(1, \infty)$ and $X$ is a UMD space. In particular, the reconstruction formula $f=\sum_{j, k}\left\langle x, \psi_{j, k}\right\rangle \psi_{j, k}$ holds, with unconditional convergence of the series involved. We show in this paper that the quoted result can be extended to redundant discrete wavelet transforms (wavelet frames). Moreover, we obtain boundedness results for certain localization operators connected with the discrete wavelet transform, where the symbols are operator-valued. For this we use an operator-valued version of the $T(1)$ theorem from [24].

Our main focus, however, will be on the continuous wavelet transform, for which we will prove a norm estimate and a reconstruction formula corresponding to the classical scalar-valued Littlewood-Paley theory (see e.g. [16]). To do this we replace the square functions appearing in the es-

2000 Mathematics Subject Classification: Primary 42C40, 42B25; Secondary 42B15, 42B20, 42B35, 46B20.

Key words and phrases: wavelets, Littlewood-Paley theory. 
timates with the generalized square functions introduced in [26]. In particular this leads to equivalent norms on Bochner spaces extending HardyLittlewood g-functions. The main tool is a recent generalization of the Mikhlin Fourier multiplier theorem to operator-valued multipliers ([37]). As a consequence we obtain results on the boundedness of localization operators for the continuous wavelet transform.

2. Definitions, notations, and main tools. Throughout this paper $X, Y$, and $Z$ are complex Banach spaces. By $\mathcal{L}(X, Y)$ we denote the space of bounded linear operators from $X$ to $Y$ and $X^{\prime}=\mathcal{L}(X, \mathbb{C})$ is the dual space of $X$. The Schwartz class $\mathcal{S}\left(\mathbb{R}^{N}, X\right)$ is the space of $X$-valued rapidly decreasing smooth functions on $\mathbb{R}^{N}$.

$\mathbb{N}=\{1,2, \ldots\}$ is the set of natural numbers and $\mathbb{N}_{0}=\{0\} \cup \mathbb{N}$. If $r$ is a positive real number, then $[r]=\max \left\{n \in \mathbb{N}_{0}: n \leq r\right\}$. We denote by $R_{*}$ the multiplicative group $\mathbb{R}_{+}$, endowed with its invariant measure $\frac{d t}{t}$.

We will need the following notions from Banach space theory.

DEFINITION 2.1.

(a) A Banach space $X$ has type $p \in[1,2]$ if there is a constant $\tau<\infty$ such that for all $n \in \mathbb{N}$ and all vectors $x_{1}, \ldots, x_{n} \in X$,

$$
\left\|\sum_{k=1}^{n} r_{k} x_{k}\right\|_{L_{2}([0,1], X)} \leq \tau\left(\sum_{k=1}^{n}\left\|x_{k}\right\|^{p}\right)^{1 / p},
$$

where $r_{k}(t)=\operatorname{sign} \sin \left(2^{k} \pi t\right)$ are the Rademacher functions on $[0,1]$.

(b) A Banach space $X$ has cotype $q \in[2, \infty]$ if there is a constant $\kappa<\infty$ such that for all $n \in \mathbb{N}$ and all vectors $x_{1}, \ldots, x_{n} \in X$,

$$
\begin{aligned}
\left(\sum_{k=1}^{n}\left\|x_{k}\right\|^{q}\right)^{1 / q} \leq \kappa\left\|\sum_{k=1}^{n} r_{k} x_{k}\right\|_{L_{2}([0,1], X)} & \text { for } q<\infty, \\
\max _{1 \leq k \leq n}\left\|x_{k}\right\| \leq \kappa\left\|\sum_{k=1}^{n} r_{k} x_{k}\right\|_{L_{2}([0,1], X)} & \text { for } q=\infty .
\end{aligned}
$$

The smallest such $\kappa$ is denoted by $C_{q}(X)$.

REMARK 2.2. Every Banach space has type 1 and the notion becomes more restrictive as $p$ increases to 2 . Similarly, every Banach space has cotype $\infty$ and the notion becomes more restrictive as $q$ decreases to 2 . By a result of Kwapień, a Banach space with both type and cotype 2 is isomorphic to a Hilbert space (see e.g. [10, Corollary 12.20]). Each closed subspace of a Banach space $X$ has the same type and cotype as $X$. A Banach space has type $p>1$ if and only if it is $B$-convex ([32], [10, Chapter 13]). Every Banach space with type $p>1$ has finite cotype (see e.g. [10, Chapters 11 and 13]). 
Definition 2.3. A Banach space $X$ has Fourier type $r \in[1,2]$ if the Fourier transform $\mathcal{F}$ defined by

$$
(\mathcal{F} f)(u)=\widehat{f}(u)=(2 \pi)^{-N / 2} \int_{\mathbb{R}^{N}} e^{-i u v} f(v) d v, \quad f \in \mathcal{S}(\mathbb{R}, X),
$$

extends to a bounded linear operator from $L_{r}(\mathbb{R}, X)$ to $L_{r^{\prime}}(\mathbb{R}, X)$, where $1 / r+1 / r^{\prime}=1$.

REMARK 2.4. The notion of Fourier type was introduced by Peetre [31]. Each Banach space has Fourier type 1 and the notion becomes more restrictive as $r$ increases to 2. A Banach space has Fourier type 2 if and only if it is isomorphic to a Hilbert space [27]. The dual space and each closed subspace of a Banach space $X$ has the same Fourier type as $X$. Bourgain $[4,5]$ has shown that each B-convex Banach space has some nontrivial Fourier type $r>1$.

Definition 2.5. A Banach space $X$ is a $U M D$ space if the Hilbert transform

$$
(\mathcal{H} f)(u)=\mathrm{PV}-\int \frac{f(v)}{u-v} d v, \quad f \in \mathcal{S}(\mathbb{R}, X),
$$

extends to a bounded linear operator on $L_{p}(\mathbb{R}, X)$ for some (and thus for each) $p \in(1, \infty)$.

REMARK 2.6. There are several equivalent definitions of UMD spaces (see [2, pp. 141-142] and the references given there). It is clear from the definition that each Hilbert space is a UMD space. The dual space and each closed subspace of a UMD space is a UMD space. A UMD space $X$ always has a uniformly convex renorming [1] and therefore is super-reflexive [12]. In particular, $\ell_{1}$ is not finitely representable in $X$. Hence $X$ is B-convex [10, Theorem 13.6]. This implies in particular that every UMD-space has type $p>1$, cotype $q<\infty$, and some nontrivial Fourier type $r>1$ (cf. Remarks 2.2 and 2.4).

REMARK 2.7. Let $(\Omega, \Sigma, \mu)$ be a $\sigma$-finite measure space, $s \in[1, \infty]$ and $X$ a Banach space. If $X$ has type $p \in[1,2]$ and cotype $q \in[2, \infty]$, then $L_{s}(\Omega, \mu, X)$ has type $\min \{p, s, 2\}$ and cotype $\max \{q, s, 2\}$ [10, Theorem 11.12]. If $X$ has Fourier type $r \in[1, \infty]$, then $L_{s}(\Omega, \mu, X)$ has Fourier type $\min \left\{r, s, s^{\prime}\right\}$ [17]. If $X$ is a UMD space and $s \in(1, \infty)$, then $L_{s}(\Omega, \mu, X)$ is a UMD space [2, p. 145].

Definition 2.8. Let $X$ be a Banach space.

(a) A function $a \in L_{\infty}\left(\mathbb{R}^{N}, X\right)$ is an atom if $a$ has its support in a cube $Q \subseteq \mathbb{R}^{N}, \int_{\mathbb{R}^{N}} a(t) d t=0$, and $\|a\|_{L_{\infty}} \leq|Q|^{-1}$. The Hardy space $H_{1}\left(\mathbb{R}^{N}, X\right)$ is the space of all $f \in L_{1}\left(\mathbb{R}^{N}, X\right)$ which can be written as $f=\sum_{k=1}^{\infty} c_{k} a_{k}$ for some sequence $\left(a_{k}\right)$ of atoms and some sequence 
$\left(c_{k}\right) \in \ell_{1}$. The norm $\|f\|_{H_{1}\left(\mathbb{R}^{N}, X\right)}$ is the infimum of $\left\|\left(c_{k}\right)\right\|_{\ell_{1}}$ over all such representations.

(b) The space of all $X$-valued functions with bounded mean oscillation is denoted by $\operatorname{BMO}\left(\mathbb{R}^{N}, X\right)$. More precisely, $\operatorname{BMO}\left(\mathbb{R}^{N}, X\right)$ contains all locally integrable functions $f: \mathbb{R}^{N} \rightarrow X$ for which the seminorm

$$
\|f\|_{\mathrm{BMO}\left(\mathbb{R}^{N}, X\right)}=\sup |Q|^{-1} \int_{Q}\left\|f(t)-f_{Q}\right\|_{X} d t
$$

is finite. Here the supremum is taken over all cubes $Q$ in $\mathbb{R}^{N}$ and $f_{Q}:=|Q|^{-1} \int_{Q} f(t) d t$ denotes the mean value of $f$ on the cube $Q$.

REMARK 2.9. If $X^{\prime}$ has the Radon-Nikodym property (in particular, if $X$ is reflexive), then (see [6])

$$
\left(H_{1}\left(\mathbb{R}^{N}, X^{\prime}\right)\right)^{\prime}=\operatorname{BMO}\left(\mathbb{R}^{N}, X\right) .
$$

In this case, the Schwartz class $\mathcal{S}\left(\mathbb{R}^{N}, X\right)$ is weak ${ }^{*}$ dense in $\operatorname{BMO}\left(\mathbb{R}^{N}, X\right)$.

Definition 2.10. Let $X, Y$ be Banach spaces, $q \in(1, \infty)$, and $M$ : $\mathbb{R}^{N} \backslash\{0\} \rightarrow \mathcal{L}(X, Y)$ be a bounded measurable function. For $f \in \mathcal{S}\left(\mathbb{R}^{N}, X\right)$ consider

$$
T f:=\mathcal{F}^{-1}[M(\cdot) \widehat{f}(\cdot)] \in L_{\infty}\left(\mathbb{R}^{N}, Y\right) .
$$

(a) $M$ is a Fourier multiplier from $L_{q}\left(\mathbb{R}^{N}, X\right)$ to $L_{q}\left(\mathbb{R}^{N}, Y\right)$ provided there is a constant $C_{q}$ so that

$$
\|T f\|_{L_{q}\left(\mathbb{R}^{N}, Y\right)} \leq C_{q}\|f\|_{L_{q}\left(\mathbb{R}^{N}, X\right)}
$$

for each $f \in \mathcal{S}\left(\mathbb{R}^{N}, X\right)$. The unique extension of $T$ to an operator in $\mathcal{L}\left(L_{q}\left(\mathbb{R}^{N}, X\right), L_{q}\left(\mathbb{R}^{N}, Y\right)\right)$ is denoted by $T_{M}$.

(b) $M$ is a Fourier multiplier from $H_{1}\left(\mathbb{R}^{N}, X\right)$ to $H_{1}\left(\mathbb{R}^{N}, Y\right)$ provided there is a constant $C_{1}$ so that

$$
\|T f\|_{H_{1}\left(\mathbb{R}^{N}, Y\right)} \leq C_{1}\|f\|_{H_{1}\left(\mathbb{R}^{N}, X\right)}
$$

for each $f \in \mathcal{S}\left(\mathbb{R}^{N}, X\right)$ with $\int_{\mathbb{R}^{N}} f(t) d t=0$. The unique extension of $T$ to a bounded linear operator from $H_{1}\left(\mathbb{R}^{N}, X\right)$ to $H_{1}\left(\mathbb{R}^{N}, Y\right)$ is denoted by $T_{M}$.

(c) $M$ is a Fourier multiplier from $\operatorname{BMO}\left(\mathbb{R}^{N}, X\right)$ to $\operatorname{BMO}\left(\mathbb{R}^{N}, Y\right)$ provided there is an operator $T_{M} \in \mathcal{L}\left(\mathrm{BMO}\left(\mathbb{R}^{N}, X\right), \mathrm{BMO}\left(\mathbb{R}^{N}, Y\right)\right)$ satisfying

$$
\begin{gathered}
T f=T_{M} f \quad \text { for all } f \in \mathcal{S}\left(\mathbb{R}^{N}, X\right), \\
T_{M} \text { is weak } \text {-to-weak }^{*} \text { continuous. }
\end{gathered}
$$

If $X, Y$ are reflexive, then $T_{M}$ is uniquely determined by $T$, since $\mathcal{S}\left(\mathbb{R}^{N}, X\right)$ is weak* dense in the space $\operatorname{BMO}\left(\mathbb{R}^{N}, X\right)$.

Now we recall the notion of R-boundedness. 
Definition 2.11. Let $X, Y$ be Banach spaces. A set of operators $\tau \subseteq$ $\mathcal{L}(X, Y)$ is called $R$-bounded if there is a constant $C<\infty$ such that for all $m \in \mathbb{N}$, all $T_{1}, \ldots, T_{m} \in \tau$ and all $x_{1}, \ldots, x_{m} \in X$ we have

$$
\left\|\sum_{k=1}^{m} r_{k} T_{k} x_{k}\right\|_{L_{2}([0,1], Y)} \leq C\left\|\sum_{k=1}^{m} r_{k} x_{k}\right\|_{L_{2}([0,1], X)},
$$

where $r_{k}(t)=\operatorname{sign} \sin \left(2^{k} \pi t\right)$ are the Rademacher functions on $[0,1]$. The infimum over all $C$ such that (2) holds is called the $R$-bound of $\tau$.

If $X$ and $Y$ are Hilbert spaces, then R-boundedness reduces to boundedness. Therefore the following Mikhlin type Fourier multiplier theorem can be seen as a generalization of a corresponding result in the Hilbert space case by Schwartz [3].

TheOREM $2.12([22,18])$. Let $X$ and $Y$ be UMD spaces with Fourier type $r \in(1,2], N \in \mathbb{N}$, and $l=[N / r]+1$. Let $M$ be an $\mathcal{L}(X, Y)$-valued function on $\mathbb{R}^{N}$. If for each multi-index $\alpha \in\{0,1\}^{N}$ with $|\alpha| \leq l$ the distributional derivatives $D^{\alpha} M$ are represented by measurable functions and the set

$$
\left\{|\xi|^{|\alpha|}\left(D^{\alpha} M\right)(\xi): \xi \in \mathbb{R}^{N} \backslash\{0\}\right\}
$$

is $R$-bounded, then $M$ is a Fourier multiplier from $E\left(\mathbb{R}^{N}, X\right)$ to $E\left(\mathbb{R}^{N}, Y\right)$, where $E \in\left\{L_{p}, H_{1}\right.$, BMO : $\left.p \in(1, \infty)\right\}$.

REMARK 2.13. Suppose the conditions of Theorem 2.12 are satisfied. Then $[M(\cdot)]^{\prime}: \mathbb{R}^{N} \backslash\{0\} \rightarrow \mathcal{L}\left(Y^{\prime}, X^{\prime}\right)$ also satisfies the conditions of Theorem 2.12 (cf. e.g. [38, Theorem 2.2.14]).

Square function estimates are a well-known tool in harmonic analysis. For instance the Littlewood-Paley characterization of $L_{p}\left(\mathbb{R}^{n}\right)$ is a characterization of that space in terms of certain square functions (cf. [16]). For our generalization of the classical Littlewood-Paley theorem to $X$-valued $L_{p}$-spaces we need the generalized square functions that were introduced in [26]. For convenience we include here the definitions and main results we will use in this paper. For a more general theory, the proofs and further references we refer to [26] (see also [32, 34]).

For the rest of this section let $\left(g_{k}\right)$ be a sequence of independent standard Gaussian random variables on a probability space $(\Gamma, \mathcal{G}, P)$.

Definition 2.14. If $H$ is a Hilbert space, $X$ is a Banach space and $u: H \rightarrow X$ is a linear operator, we define

$$
\|u\|_{\gamma(H, X)}:=\sup \left(\mathbb{E}\left\|\sum_{k} g_{k} u\left(e_{k}\right)\right\|^{2}\right)^{1 / 2}
$$

where the sup is taken over all finite orthonormal systems $\left(e_{k}\right)$ in $H$. More- 
over, we define $\gamma(H, X)$ as the completion of the finite rank operators in $\mathcal{L}(H, X)$ with respect to $\left.\|\cdot\|_{\gamma(H, X)}{ }^{1}\right)$.

By definition, $\left(\gamma(H, X),\|\cdot\|_{\gamma(H, X)}\right)$ is a Banach space. It is even a Banach ideal ([26, Prop. 4.3]; see also [15, 32, 34]).

Remark 2.15. If $X$ does not contain $c_{0}$ and $H$ is separable, then

$$
\|u\|_{\gamma(H, X)}=\left(\mathbb{E}\left\|\sum_{k=1}^{\infty} g_{k} u\left(e_{k}\right)\right\|^{2}\right)^{1 / 2},
$$

independently of the chosen orthonormal basis $\left\{e_{k}\right\}$ of $H$ (cf. $[26,28]$ ). In particular, $\gamma(H, X)$ is isometrically embedded into $L_{2}(\Gamma, P, X)$. This means that the properties UMD, Fourier type, type and cotype lift from $X$ to $\gamma(H, X)$, since they lift to $L_{2}(\Gamma, P, X)$ (cf. Remark 2.7).

Now let $H=L_{2}(\Omega, \mu)$, where $(\Omega, \Sigma, \mu)$ is a $\sigma$-finite measure space with countably generated $\sigma$-algebra $\Sigma$. Then $H$ is separable (cf. [8, p. 110]). If $f: \Omega \rightarrow X$ is a $\mu$-measurable function that is weakly in $H$ (i.e., $x^{\prime} \circ f \in H$ for each $x^{\prime} \in X^{\prime}$ ), then we can define a bounded linear operator $u_{f}: H \rightarrow X$ by the formula

$$
\left\langle x^{\prime}, u_{f} h\right\rangle=\int_{\Omega}\left\langle x^{\prime}, f(\omega)\right\rangle h(\omega) d \mu(\omega), \quad x^{\prime} \in X^{\prime}, h \in H .
$$

For a proof that $u_{f}$ is indeed bounded from $H$ to $X$ see [26]. We write $f \in \gamma(\Omega, \mu, X)$ if $u_{f} \in \gamma(H, X)$, and set

$$
\|f\|_{\gamma(\Omega, \mu, X)}:=\left\|u_{f}\right\|_{\gamma(H, X)} .
$$

If $\Omega=\mathbb{Z}$ with the counting measure, i.e. $H=\ell_{2}$, then we use the notation $\gamma(\mathbb{Z}, X)$.

REMARK 2.16. If $X$ is a Banach space not containing $c_{0}$, then the set $\left\{u_{f}: f \in \gamma(\Omega, \mu, X)\right\}$ is a dense subset of $\gamma(H, X)$. Therefore one may consider the operator space $\gamma(H, X)$ as the completion of the function space $\gamma(\Omega, \mu, X)$ (cf. [26, Remark 4.7]).

REMARK 2.17. If $X$ has type $>1$, then $\gamma\left(H, X^{\prime}\right)$ is a closed subspace of $\gamma(H, X)^{\prime}$, the dual of $\gamma(H, X)$ with respect to trace duality ([26, Section 5]). If $h \in H, x^{\prime} \in X^{\prime}$, and $y \in \gamma(H, X)$, then

$$
\left\langle h \otimes x^{\prime}, y\right\rangle_{\gamma}=\left\langle x^{\prime}, y(h)\right\rangle_{X} .
$$

REMARK 2.18. If $X$ is some $L_{q}$-space with $1<q<\infty$, then $\|\cdot\|_{\gamma(\Omega, \mu, X)}$ is equivalent to the classical square function norm:

$$
\|f\|_{\gamma\left(\Omega, \mu, L_{q}\right)} \sim\left\|\left(\int_{\Omega}|f(\omega)|^{2} d \mu(\omega)\right)^{1 / 2}\right\|_{L_{q}} .
$$

$\left({ }^{1}\right)$ In the literature, the spaces $\gamma(H, X)$ are also denoted by $\ell(H, X)$. 
3. An estimate for random series. The main condition in the multiplier theorem 2.12 is the R-boundedness of the multiplier function and its derivatives. To check this in our setting we need an estimate of the following type. If $x_{k} \in X$ and $m_{k} \in L_{2}(\Omega, \mu)$, we would like to have

$$
\left\|\sum_{k=1}^{n} r_{k}(\cdot) m_{k} \otimes x_{k}\right\|_{L_{2}([0,1], \gamma(\Omega, \mu, X))} \lesssim \max _{k \leq n}\left\|m_{k}\right\|_{2}\left\|\sum_{k=1}^{n} r_{k}(\cdot) x_{k}\right\|_{L_{2}([0,1], X)},
$$

where the constant in the estimate has to be independent of the choice of $n$, $x_{k}$ and $m_{k}$, but may depend on the Banach space $X$. If $X$ is some $L_{q}$-space with $q \in[1, \infty)$, this estimate is a simple consequence of Remark 2.18, Khinchin's (respectively Kahane's) inequality, and the contraction principle (see [25]). To prove the desired estimate in a more general setting we will use part (b) of the following lemma, which should be of some interest in itself.

In this section $X$ is always a complex Banach space.

Lemma 3.1. For $k \in \mathbb{N}$ let $\sigma_{k}$ be positve real numbers and $g_{k}$ (not necessarily independent) Gaussian random variables on some probability space $(\Gamma, \mathcal{G}, P)$ with mean 0 and variance $\sigma_{k}^{2}$.

(a) There is a constant $c>0$ such that for all $n \in \mathbb{N}$ and all vectors $x_{1}, \ldots, x_{n} \in X$

$$
c \min _{k \leq n} \sigma_{k}\left\|\sum_{k=1}^{n} r_{k}(\cdot) x_{k}\right\|_{L_{2}([0,1], X)} \leq\left(\mathbb{E}\left\|\sum_{k=1}^{n} g_{k} r_{k}(\cdot) x_{k}\right\|_{L_{2}([0,1], X)}^{2}\right)^{1 / 2} .
$$

(b) If $X$ has finite cotype, then there is a constant $C<\infty$ such that for all $n \in \mathbb{N}$ and all vectors $x_{1}, \ldots, x_{n} \in X$,

$$
\left(\mathbb{E}\left\|\sum_{k=1}^{n} r_{k}(\cdot) g_{k} x_{k}\right\|_{L_{2}([0,1], X)}^{2}\right)^{1 / 2} \leq C \max _{k \leq n} \sigma_{k}\left\|\sum_{k=1}^{n} r_{k}(\cdot) x_{k}\right\|_{L_{2}([0,1], X)} .
$$

REMARK 3.2. For independent Gaussian random variables $g_{k}$, Lemma 3.1 follows from well-known results on Gaussian and Rademacher sums (see e.g. Proposition 12.11 and Theorem 12.27 in [10], cf. also [34]), because in this case

$$
\mathbb{E}\left\|\sum_{k=1}^{n} r_{k}(\cdot) g_{k} x_{k}\right\|_{L_{2}([0,1], X)}^{2}=\mathbb{E}\left\|\sum_{k=1}^{n} g_{k} x_{k}\right\|^{2}
$$

(cf. [10, Lemma 11.2]). If the $g_{k}$ are not independent, we cannot use the known results directly, but the proofs can be adapted to this more general setting.

Proof of Lemma 3.1. If $g$ is a Gaussian random variable with mean 0 and variance $\sigma^{2}$ for some $\sigma>0$, then the $r$ th moment of $g$ is

$$
\left(\int_{\Gamma}|g(\omega)|^{r} d P(\omega)\right)^{1 / r}=\sigma m_{r}
$$

where $m_{r}$ is the $r$ th moment of a standard Gaussian random variable. 
(a) Using Kahane's contraction principle and (4), we obtain

$$
\begin{aligned}
& \left\|\sum_{k=1}^{n} r_{k}(\cdot) x_{k}\right\|_{L_{2}([0,1], X)} \\
& \quad \leq \max _{k \leq n}\left[\left(m_{1} \sigma_{k}\right)^{-1}\right]\left\|\sum_{k=1}^{n} r_{k}(\cdot) \int_{\Gamma}\left|g_{k}(\omega)\right| d P(\omega) x_{k}\right\|_{L_{2}([0,1], X)} .
\end{aligned}
$$

By Minkowski's and Hölder's inequalities

$$
\begin{aligned}
m_{1} \min _{k \leq n} \sigma_{k}\left\|\sum_{k=1}^{n} r_{k}(\cdot) x_{k}\right\|_{L_{2}([0,1], X)} & \leq\left(\int\left\|\sum_{\Gamma=1}^{n} r_{k}(\cdot)\left|g_{k}(\omega)\right| x_{k}\right\|_{L_{2}([0,1], X)}^{2} d P(\omega)\right)^{1 / 2} \\
& =\left(\int\left\|\sum_{\Gamma=1}^{n} r_{k}(\cdot) g_{k}(\omega) x_{k}\right\|_{L_{2}([0,1], X)}^{2} d P(\omega)\right)^{1 / 2} .
\end{aligned}
$$

In the last step we used the fact that, for any choice of $\varepsilon_{k}= \pm 1$,

$$
\left\|\sum_{k=1}^{n} r_{k}(\cdot) y_{k}\right\|_{L_{2}([0,1], X)}=\left\|\sum_{k=1}^{n} r_{k}(\cdot) \varepsilon_{k} y_{k}\right\|_{L_{2}([0,1], X)}
$$

(cf. [10, Lemma 11.2]).

(b) Fix $x_{1}, \ldots, x_{n} \in X$. Define $u \in \mathcal{L}\left(\ell_{\infty}^{n}, L_{2}([0,1], X)\right)$ by $u\left(e_{k}\right)=$ $r_{k}(\cdot) x_{k}$ for $k=1, \ldots, n$. Here $e_{k}$ denotes the standard unit vector in $\ell_{\infty}^{n}$, the $n$-dimensional space $\mathbb{R}^{n}$ with sup-norm. Observe that by Kahane's contraction principle

$$
\|u\|=\left\|\sum_{k=1}^{n} r_{k}(\cdot) x_{k}\right\|_{L_{2}([0,1], X)} .
$$

Using our assumption that $X$ has cotype $q \in[2, \infty)$ and Pietsch's domination theorem ([10, Theorem 2.12]) one can show that there are nonnegative $\lambda_{k}$ with $\sum_{k=1}^{n} \lambda_{k}=1$ such that

$$
\|u(b)\|_{L_{2}([0,1], X)} \leq C_{q}(X)\|u\|\left(\sum_{k=1}^{n} \lambda_{k}\left|b_{k}\right|^{r}\right)^{1 / r} \quad \text { for all } b=\left(b_{k}\right) \in \ell_{\infty}^{n}
$$

where $r$ is some real number $>q$ (cf. the proof of Theorem 12.27 in [10]). Therefore, with our notation above,

$$
\begin{aligned}
\left(\int_{\Gamma}\left\|\sum_{k=1}^{n} r_{k}(\cdot) g_{k}(\omega) x_{k}\right\|_{L_{2}([0,1], X)}^{2}\right. & d P(\omega))^{1 / 2} \\
& =\left(\int_{\Gamma}\left\|u\left(\left(g_{k}(\omega)\right)_{k}\right)\right\|_{L_{2}([0,1], X)}^{2} d P(\omega)\right)^{1 / 2}
\end{aligned}
$$




$$
\leq C_{q}(X)\|u\|\left(\int_{\Gamma}\left(\sum_{k=1}^{n} \lambda_{k}\left|g_{k}(\omega)\right|^{r}\right)^{2 / r} d P(\omega)\right)^{1 / 2} .
$$

By Hölder's inequality and (4),

$$
\begin{aligned}
\left(\int_{\Gamma}\left(\sum_{k=1}^{n} \lambda_{k}\left|g_{k}(\omega)\right|^{r}\right)^{2 / r} d P(\omega)\right)^{1 / 2} & \leq\left(\sum_{k=1}^{n} \lambda_{k} \int_{\Gamma}\left|g_{k}(\omega)\right|^{r} d P(\omega)\right)^{1 / r} \\
& =\left(\sum_{k=1}^{n} \lambda_{k} \sigma_{k}^{r}\right)^{1 / r} m_{r} \leq m_{r} \max _{k \leq n} \sigma_{k} .
\end{aligned}
$$

Together with (5) this completes the proof.

REMARK 3.3. The proof of Lemma 3.1 shows that we can choose $c=m_{1}$ and $C=C_{q}(X) m_{r}$, provided $X$ has cotype $q$ and $2 \leq q<r<\infty$.

COROLlary 3.4. Let $\left(g_{j}\right)$ be a sequence of independent standard Gaussian variables on some probability space. If $X$ has finite cotype, then there is a constant $C<\infty$ such that for all $m, n \in \mathbb{N}$, all vectors $x_{1}, \ldots, x_{n} \in X$ and all complex numbers $a_{j k}(j=1, \ldots, m, k=1, \ldots, n)$, we have the estimate

$$
\begin{aligned}
\left(\mathbb{E}\left\|\sum_{k=1}^{n} r_{k}(\cdot) \sum_{j=1}^{m} g_{j} a_{j k} x_{k}\right\|_{L_{2}([0,1], X)}^{2}\right)^{1 / 2} & \\
& \leq C \max _{k \leq n}\left(\sum_{j=1}^{m}\left|a_{j k}\right|^{2}\right)^{1 / 2}\left\|\sum_{k=1}^{n} r_{k}(\cdot) x_{k}\right\|_{L_{2}([0,1], X)} .
\end{aligned}
$$

Proof. Suppose first that the $a_{j k}$ are real. Since $\left(g_{j}\right)$ are independent standard Gaussian variables, $\widetilde{g}_{k}=\sum_{j=1}^{m} g_{j} a_{j k}$ is a Gaussian variable with mean 0 and variance $\sigma_{k}^{2}=\sum_{j=1}^{m}\left|a_{j k}\right|^{2}$ for each $k$ (cf. [10, p. 238]). (Note that $\left(\widetilde{g}_{k}\right)$ do not have to be independent any more.) Now we can apply Lemma 3.1(b).

If the $a_{j k}$ are complex numbers, we treat the real and imaginary parts separately.

REMARK 3.5. It is also possible to obtain a reverse inequality, without any assumptions on the geometry of the Banach space $X$. Namely, let $\left(g_{j}\right)$ be a sequence of independent standard Gaussian variables on some probability space. Then there is a constant $c>0$ such that for all $m, n \in \mathbb{N}$, all vectors $x_{1}, \ldots, x_{n} \in X$ and all complex numbers $a_{j k}(j=1, \ldots, m, k=1, \ldots, n)$,

$$
\begin{aligned}
c \min _{k \leq n}\left(\sum_{j=1}^{m}\left|a_{j k}\right|^{2}\right)^{1 / 2} \| \sum_{k=1}^{n} r_{k}(\cdot) & x_{k} \|_{L_{2}([0,1], X)} \\
& \leq\left(\mathbb{E}\left\|\sum_{k=1}^{n} r_{k}(\cdot) \sum_{j=1}^{m} g_{j} a_{j k} x_{k}\right\|_{L_{2}([0,1], X)}^{2}\right)^{1 / 2} .
\end{aligned}
$$


If the $a_{j k}$ are real numbers, this is just a simple consequence of Lemma 3.1(a) by the same argument as in the proof of Corollary 3.4. In the complex case we observe that (with the notation from the proof above) $2^{-1 / 2} \sigma_{k} m_{1} \leq$ $\int_{\Gamma}\left|\widetilde{g}_{k}(\omega)\right| d P(\omega) \leq 2 \sigma_{k} m_{1}$. Now a slight modification of the argument in the proof of Lemma 3.1 gives the desired result. For further details see [20, Sect. 3.7].

Now we come back to the estimate we stated at the beginning of this section. It now follows easily from what we did so far.

Corollary 3.6. Let $X$ be a Banach space of cotype $q \in[2, \infty)$ and $(\Omega, \Sigma, \mu)$ a $\sigma$-finite measure space with countably generated $\sigma$-algebra $\Sigma$. Then there is a constant $C<\infty$ such that for all $n \in \mathbb{N}$, all $m_{1}, \ldots, m_{n} \in$ $L_{2}(\Omega, \mu)$ and all $x_{1}, \ldots, x_{n} \in X$,

$$
\begin{aligned}
& \left(\int_{0}^{1}\left\|\sum_{k=1}^{n} r_{k}(t) m_{k}(\cdot) x_{k}\right\|_{\gamma(\Omega, \mu, X)}^{2} d t\right)^{1 / 2} \\
& \quad \leq C \max _{k \leq n}\left\|m_{k}\right\|_{2}\left(\int_{0}^{1}\left\|\sum_{k=1}^{n} r_{k}(t) x_{k}\right\|_{X}^{2} d t\right)^{1 / 2} .
\end{aligned}
$$

Proof. Choose an orthonormal basis $\left\{e_{j}\right\}_{j=1}^{\infty}$ in $L_{2}(\Omega, \mu)$ and $m \in \mathbb{N}$ such that

$$
a_{j k}:=\int_{\Omega} m_{k}(\omega) e_{j}(\omega) d \mu(\omega)=0 \quad \text { for all } j>m \text { and all } k .
$$

Then $\left\|m_{k}\right\|_{2}^{2}=\sum_{j=1}^{m}\left|a_{j k}\right|^{2}$, and by Corollary 3.4,

$$
\begin{aligned}
\left(\int_{0}^{1}\left\|\sum_{k=1}^{n} r_{k}(t) m_{k}(\cdot) x_{k}\right\|_{\gamma(\Omega, \mu, X)}^{2} d t\right)^{1 / 2} & \left(\int_{0}^{1} \mathbb{E}\left\|\sum_{k=1}^{n} r_{k}(t) \sum_{j=1}^{\infty} g_{j} \int_{\Omega} m_{k}(\omega) e_{j}(\omega) d \mu(\omega) x_{k}\right\|_{X}^{2}\right)^{1 / 2} \\
& =\left(\mathbb{E}\left\|\sum_{k=1}^{n} r_{k}(\cdot) \sum_{j=1}^{m} g_{j} a_{j k} x_{k}\right\|_{L_{2}([0,1], X)}^{2}\right)^{1 / 2} \\
& \leq C \sup _{k}\left(\sum_{j=1}^{m}\left|a_{j k}\right|^{2}\right)^{1 / 2}\left\|\sum_{k=1}^{n} r_{k}(\cdot) x_{k}\right\|_{L_{2}([0,1], X)}
\end{aligned}
$$

REMARK 3.7. It is clear from Remark 3.5 and the argument in the proof above that we also have a reverse inequality, involving the minimum over the $L_{2}$-norms of the $m_{k}$ instead of the maximum. Again we do not need any assumptions on the geometry of the Banach space for this reverse estimate. 
4. The continuous wavelet transform. Let $X$ be a UMD space with Fourier type $r \in(1,2], N \in \mathbb{N}$ and $l:=[N / r]+1$. We will need the following two assumptions on the wavelet $\psi \in L_{2}\left(\mathbb{R}^{N}\right)$ :

(C1) For all $\alpha \in\{0,1\}^{N}$ with $|\alpha| \leq l$, the distributional derivatives $D^{\alpha} \widehat{\psi}$ are represented by measurable functions and

$$
C_{\alpha}(\psi):=\sup _{|\xi|=1}\left(\int_{0}^{\infty} t^{2|\alpha|}\left|\left(D^{\alpha} \widehat{\psi}\right)(t \xi)\right|^{2} \frac{d t}{t}\right)^{1 / 2}<\infty
$$

$(\mathrm{C} 2) c_{0}(\psi):=\inf _{|\xi|=1}\left(\int_{0}^{\infty}|\widehat{\psi}(t \xi)|^{2} \frac{d t}{t}\right)^{1 / 2}>0$.

For $t \in \mathbb{R} \backslash\{0\}$ we write $\psi_{t}$ for the function defined by $\psi_{t}(s)=|t|^{-N} \psi\left(t^{-1} s\right)$, $s \in \mathbb{R}^{N}$.

Definition 4.1. The continuous wavelet transform $\mathcal{W}_{\psi} f$ of a function $f \in \mathcal{S}\left(\mathbb{R}^{N}, X\right)$ with respect to the wavelet $\psi$ is given by

$$
\left(\mathcal{W}_{\psi} f\right)(t, s):=\left(\psi_{t} * f\right)(s)=\int_{\mathbb{R}^{N}} t^{-N} \psi\left(\frac{s-u}{t}\right) f(u) d u, \quad t>0, s \in \mathbb{R}^{N} .
$$

It is the goal of this section to prove that one can characterize the function spaces $L_{p}\left(\mathbb{R}^{N}, X\right)(1<p<\infty), H_{1}\left(\mathbb{R}^{N}, X\right)$, and $\mathrm{BMO}\left(\mathbb{R}^{N}, X\right)$ in terms of the continuous wavelet transform. In the following theorem $E\left(\mathbb{R}^{N}, X\right)$ stands for one of these spaces.

TheOREM 4.2. Let $X$ be a UMD space with Fourier type $r \in(1,2]$, $p \in(1, \infty), N \in \mathbb{N}$, and $l:=[N / r]+1$.

(a) If $\psi \in L_{2}\left(\mathbb{R}^{N}\right)$ satisfies $(\mathrm{C} 1)$, then $\mathcal{W}_{\psi}$ defines a bounded linear operator from $E\left(\mathbb{R}^{N}, X\right)$ to $E\left(\mathbb{R}^{N}, \gamma\left(\mathbb{R}_{*}, X\right)\right)$, where $E \in\left\{L_{p}, H_{1}\right.$, BMO $\}$.

(b) If $\psi \in L_{2}\left(\mathbb{R}^{N}\right)$ satisfies $(\mathrm{C} 1)$ and $(\mathrm{C} 2)$, then

$$
\left\|\mathcal{W}_{\psi} f\right\|_{E\left(\mathbb{R}^{N}, \gamma\left(\mathbb{R}_{*}, X\right)\right)} \sim\|f\|_{E\left(\mathbb{R}^{N}, X\right)}
$$

for all $f \in E\left(\mathbb{R}^{N}, X\right)$ with $E \in\left\{L_{p}, H_{1}, \mathrm{BMO}\right\}$.

Proof. (a) The main idea of the proof is to define the operator-valued multiplier

$$
\begin{aligned}
& M_{\psi}: \mathbb{R}^{N} \backslash\{0\} \rightarrow \mathcal{L}(X, Y), \\
& M_{\psi}(\xi) x=\left[t \mapsto \widehat{\psi}_{t}(\xi) x\right]=[t \mapsto \widehat{\psi}(t \xi) x],
\end{aligned}
$$

and to apply the multiplier theorem 2.12. Here $Y$ is the completion of $\gamma\left(\mathbb{R}_{*}, X\right)$, which can be identified with $\gamma\left(L_{2}\left(\mathbb{R}_{*}\right), X\right)$ (cf. Remark 2.16). 
First we make the following two observations:

(1) The map $J: L_{2}\left(\mathbb{R}_{*}\right) \rightarrow \mathcal{L}(X, Y)$ given by $(J h) x=[t \mapsto h(t) x]$ is an isometric embedding since

$$
\|J h\|=\sup _{\|x\|=1}\|(J h) x\|_{Y}=\sup _{\|x\|=1}\|h(\cdot) x\|_{Y}=\|h\|_{L_{2}\left(\mathbb{R}_{*}\right)} .
$$

This implies in particular that, for each $\xi \in \mathbb{R}^{N} \backslash\{0\}, M_{\psi}(\xi)=$ $J[\widehat{\psi}(\cdot \xi)]$ is a bounded linear operator from $X$ to $Y$. So $M_{\psi}$ is welldefined.

(2) If $m: \mathbb{R}^{N} \backslash\{0\} \rightarrow \mathbb{C}$ is measurable with $m(\cdot \xi) \in L_{2}\left(\mathbb{R}_{*}\right)$ for all $\xi \in \mathbb{R}^{N} \backslash\{0\}$, then $\xi \mapsto m(\cdot \xi)$ is measurable as a function from $\mathbb{R}^{N} \backslash\{0\}$ to $L_{2}\left(\mathbb{R}_{*}\right)$. This follows from Pettis' theorem, since $L_{2}\left(\mathbb{R}_{*}\right)$ is separable and $\xi \mapsto m(\cdot \xi)$ is weakly measurable.

By our assumption (C1) and observation (2), the distributional derivatives of order $\alpha$ with $|\alpha| \leq l$ of the function $\xi \mapsto \widehat{\psi}(\cdot \xi)$, given by $\xi \mapsto[t \mapsto$ $\left.t^{|\alpha|}\left(D^{\alpha} \widehat{\psi}\right)(t \xi)\right]$, are measurable functions from $\mathbb{R}^{N} \backslash\{0\}$ to $L_{2}\left(\mathbb{R}_{*}\right)$. Using observation (1) we deduce that, for $|\alpha| \leq l$, the distributional derivatives $D^{\alpha} M_{\psi}$ have the form $\left(D^{\alpha} M_{\psi}\right)(\xi) x=\left[t \mapsto t^{|\alpha|}\left(D^{\alpha} \widehat{\psi}\right)(t \xi) x\right]$ and are measurable functions from $\mathbb{R}^{N} \backslash\{0\}$ to $\mathcal{L}(X, Y)$.

Fix $\alpha \in \mathbb{N}_{0}$ with $|\alpha| \leq l$. Then the set $\left\{|\xi|^{|\alpha|}\left(D^{\alpha} M_{\psi}\right)(\xi): \xi \in \mathbb{R}^{N} \backslash\{0\}\right\}$ is R-bounded for $|\alpha| \leq l$. Indeed, for all $m \in \mathbb{N}$, all $\xi_{k} \in \mathbb{R}^{N} \backslash\{0\}$ and all $x_{k} \in X, k=1, \ldots, m$,

$$
\begin{aligned}
& \left\|\sum_{k=1}^{m} r_{k}\left|\xi_{k}\right|^{|\alpha|} D^{\alpha} M_{\psi}\left(\xi_{k}\right) x_{k}\right\|_{L_{2}([0,1], Y)} \\
& \quad \leq c_{X} \sup _{k}\left(\int_{0}^{\infty}\left|t \xi_{k}\right|^{2|\alpha|}\left|\left(D^{\alpha} \widehat{\psi}\right)\left(t \xi_{k}\right)\right|^{2} \frac{d t}{t}\right)^{1 / 2}\left\|\sum_{k=1}^{m} r_{k} x_{k}\right\|_{L_{2}([0,1], X)} \\
& \quad \leq c_{X} C_{\alpha}(\psi)\left\|\sum_{k=1}^{m} r_{k} x_{k}\right\|_{L_{2}([0,1], X)}
\end{aligned}
$$

by Corollary 3.6 and (C1). Now Theorem 2.12 implies that $M_{\psi}$ is a Fourier multiplier from $E\left(\mathbb{R}^{N}, X\right)$ to $E\left(\mathbb{R}^{N}, Y\right)$, where $E \in\left\{L_{p}, H_{1}, \mathrm{BMO}\right\}$. Since for $f \in \mathcal{S}\left(\mathbb{R}^{N}, X\right)$ we have

$$
\left(\mathcal{W}_{\psi} f\right)(\cdot, s)=(\psi . * f)(s)=(2 \pi)^{-N / 2}\left(\mathcal{F}^{-1}\left[M_{\psi} \widehat{f}\right]\right)(s),
$$

this completes the proof of part (a) of the theorem.

(b) Observe first, that if $\psi \in L_{2}\left(\mathbb{R}^{N}\right)$ satisfies (C1) and (C2), then $\varphi$ defined by

$$
\widehat{\varphi}(\xi):=\left(\int_{0}^{\infty}|\widehat{\psi}(t \xi)|^{2} \frac{d t}{t}\right)^{-1} \bar{\psi}(\xi), \quad \xi \in \mathbb{R}^{N}
$$


also satisfies (C1) and (C2). Indeed, using our assumptions on $\psi$ we see immediately that $\varphi \in L_{2}\left(\mathbb{R}^{N}\right)$ and $\varphi$ satisfies (C2). To show that $\varphi$ satisfies (C1), we look at $h$ defined by $h(\xi)=\int_{0}^{\infty}|\widehat{\psi}(t \xi)|^{2} d t / t, \xi \in \mathbb{R}^{N} \backslash\{0\}$. By our assumptions on $\psi$, it follows that $h$ and its distributional derivatives of order $\leq l$ are bounded. Moreover, $1 / h$ is bounded and therefore has bounded distributional derivatives of order $\leq l$. Using Leibniz' formula and the fact that $h$ is positively homogeneous of degree 0 (i.e. $h(t \xi)=h(\xi)$ for all $t>0$ and all $\xi \in \mathbb{R}^{N} \backslash\{0\}$ ), we obtain

$$
(t \xi)^{\alpha}\left(D^{\alpha} \widehat{\varphi}\right)(t \xi)=\sum_{\beta \leq \alpha}\left(\begin{array}{l}
\alpha \\
\beta
\end{array}\right) \xi^{\alpha-\beta}\left(D^{\alpha-\beta} \frac{1}{h}\right)(\xi)(t \xi)^{\beta}\left(D^{\beta} \widehat{\psi}\right)(t \xi) .
$$

Hence $\varphi$ satisfies (C2).

For $\xi \in \mathbb{R}^{N} \backslash\{0\}$ and $y \in Y=\gamma\left(L_{2}\left(\mathbb{R}_{*}\right), X\right)$ we define

$$
N_{\varphi}(\xi) y:=y(\widehat{\varphi}(\cdot \xi)) \text {. }
$$

Then $N_{\varphi}(\xi) \in \mathcal{L}(Y, X)$, since

$$
\left\|N_{\varphi}(\xi) y\right\|_{X} \leq\|y\|_{\mathcal{L}\left(L_{2}\left(\mathbb{R}_{*}\right), X\right)}\|\widehat{\varphi}(\cdot \xi)\|_{L_{2}\left(\mathbb{R}_{*}\right)} \leq C_{0}\|y\|_{Y} .
$$

Now let $M_{\varphi}: \mathbb{R}^{N} \backslash\{0\} \rightarrow \mathcal{L}\left(X^{\prime}, \gamma\left(L_{2}\left(\mathbb{R}_{*}\right), X^{\prime}\right)\right)$ be defined as in (7). From Remark 2.17 we know that $\gamma\left(L_{2}\left(\mathbb{R}_{*}\right), X^{\prime}\right)$ is a closed subspace of $Y^{\prime}$. Moreover, by (3), we obtain for $x^{\prime} \in X^{\prime}$ and $\xi \in \mathbb{R}^{N} \backslash\{0\}$ the duality relation

$$
\left\langle M_{\varphi}(\xi) x^{\prime}, y\right\rangle_{\gamma}=\left\langle\widehat{\varphi}(\cdot \xi) x^{\prime}, y\right\rangle_{\gamma}=\left\langle x^{\prime}, y(\widehat{\varphi}(\cdot \xi))\right\rangle_{X}=\left\langle x^{\prime}, N_{\varphi}(\xi) y\right\rangle_{X} .
$$

Therefore, $N_{\varphi}(\xi)=M_{\varphi}(\xi)^{\prime}$ and $N_{\varphi}$ satisfies the conditions of Theorem 2.12, since $M_{\varphi}$ does (cf. Remark 2.13). Thus $N_{\varphi}$ is a Fourier multiplier from $E\left(\mathbb{R}^{N}, Y\right)$ to $E\left(\mathbb{R}^{N}, X\right)$, where $E \in\left\{L_{p}, H_{1}, \mathrm{BMO}\right\}$. But

$$
\begin{aligned}
\left\langle x^{\prime}, N_{\varphi}(\xi) M_{\psi}(\xi) x\right\rangle_{X} & =\left\langle x^{\prime}, u_{M_{\psi}(\xi) x}(\widehat{\varphi}(\cdot \xi))\right\rangle_{X}=\int_{0}^{\infty} \widehat{\varphi}(t \xi)\left\langle x^{\prime}, \widehat{\psi}(t \xi) x\right\rangle \frac{d t}{t} \\
& =\int_{0}^{\infty} \widehat{\varphi}(t \xi) \widehat{\psi}(t \xi) \frac{d t}{t}\left\langle x^{\prime}, x\right\rangle=\left\langle x^{\prime}, x\right\rangle .
\end{aligned}
$$

So $N_{\varphi}(\xi) M_{\psi}(\xi)=\operatorname{Id}_{X}$ for all $\xi \in \mathbb{R}^{N} \backslash\{0\}$.

REMARK 4.3 (Classical square functions). (a) If $X=L_{q}(\Omega, \mu)$ for some $q \in(1, \infty)$ and a $\sigma$-finite measure space $(\Omega, \Sigma, \mu)$, then the norm equivalence in Theorem 4.2 reads (cf. Remark 2.18)

$$
\left\|\left(\int_{0}^{\infty}\left|\left(\mathcal{W}_{\psi} f\right)(t, \cdot)\right|^{2} \frac{d t}{t}\right)^{1 / 2}\right\|_{L_{p}\left(\mathbb{R}^{N}, X\right)} \sim\|f\|_{L_{p}\left(\mathbb{R}^{N}, X\right)} .
$$

(b) Let $P(x)=c_{n}\left(1+|x|^{2}\right)^{-(n+1) / 2}$, where $c_{n}>0$ is such that $\widehat{P}(\xi)=$ $e^{-|\xi|}$. Then $P_{t}(x)=t^{-n} P(x / t)$ is the Poisson kernel. Let $\psi(x):=\left.\frac{d}{d t} P_{t}(x)\right|_{t=1}$. Then $\psi$ satisfies conditions $(\mathrm{C} 1)$ and $(\mathrm{C} 2)$. Indeed, $\widehat{\psi}(\xi)=\left.\frac{d}{d t} \widehat{P}_{t}(\xi)\right|_{t=1}=$ 
$\left.\frac{d}{d t} \widehat{P}(t \xi)\right|_{t=1}=-|\xi| e^{-|\xi|}$. A direct calculation of the derivatives completes the argument.

In the same way one can show that $\varphi(x)=\left.\frac{d}{d t} G_{\sqrt{t}}(x)\right|_{t=1}$ satisfies $(\mathrm{C} 1)$ and (C2). Here $G_{\sqrt{t}}$ denotes the Gaussian kernel with $G(x)=e^{-|x|^{2} / 2}$.

With these choices of $\varphi$ and $\psi$ we obtain vector-valued analogues of the g-function of Littlewood-Paley ([33], see also [36]).

REMARK 4.4 (Reconstruction formula). Suppose $\psi, \varphi \in L_{2}(\mathbb{R})$ satisfy $(\mathrm{C} 1)$ and $\int_{\mathbb{R}} \widehat{\varphi}(t \xi) \widehat{\psi}(t \xi) \frac{d t}{t}=1$ for almost all $\xi \in \mathbb{R}^{N} \backslash\{0\}$. Let us denote by $\mathcal{M}_{\varphi}$ the Fourier multiplier operator associated with the multiplier function $N_{\varphi}$ defined in (8). Then the proof of part (b) of Theorem 4.2 implies that $\mathcal{M}_{\varphi}$ is a bounded linear operator from $E\left(\mathbb{R}^{N}, Y\right)$ to $E\left(\mathbb{R}^{N}, X\right)$ and that

$$
f=\mathcal{M}_{\varphi} \mathcal{W}_{\psi} f
$$

for all $f \in E\left(\mathbb{R}^{N}, X\right)$. If $f \in \mathcal{S}\left(\mathbb{R}^{N}, X\right)$, this reconstruction formula can be written as

$$
f=(2 \pi)^{-N} \int_{0}^{\infty} \varphi_{t} * \psi_{t} * f \frac{d t}{t} .
$$

Indeed, for $g \in \mathcal{S}\left(\mathbb{R}^{N}, X^{\prime}\right)$, Fubini's theorem yields

$$
\begin{aligned}
\int_{\mathbb{R}^{N}}\langle g(s), f(s)\rangle_{X} d s & =\int_{\mathbb{R}^{N}} \int_{0}^{\infty} \widehat{\varphi}(t \xi) \widehat{\psi}(t \xi) \frac{d t}{t}\langle\widehat{g}(-\xi), \widehat{f}(\xi)\rangle_{X} d \xi \\
& =\int_{0}^{\infty} \int_{\mathbb{R}^{N}}\langle\widehat{g}(-\xi), \widehat{\varphi}(t \xi) \widehat{\psi}(t \xi) \widehat{f}(\xi)\rangle_{X} d \xi \frac{d t}{t} \\
& =\int_{0}^{\infty} \int_{\mathbb{R}^{N}}\left\langle g(s),(2 \pi)^{-N}\left(\varphi_{t} * \psi_{t} * f\right)(s)\right\rangle_{X} d s \frac{d t}{t} \\
& =\lim _{\substack{\varepsilon \rightarrow 0 \\
R \rightarrow \infty}} \int_{\mathbb{R}^{N}}\left\langle g(s),(2 \pi)^{-N} \int_{\varepsilon}^{R}\left(\varphi_{t} * \psi_{t} * f\right)(s) \frac{d t}{t}\right\rangle_{X} d s .
\end{aligned}
$$

(Observe that $\left\|\varphi_{t} * \psi_{t} * f\right\|_{L_{\infty}} \leq t^{-1}\|\varphi\|_{2}\|\psi\|_{2}\|f\|_{1}$.) Now it is not hard to show that for each $f \in \mathcal{S}\left(\mathbb{R}^{N}, X\right) \cap E\left(\mathbb{R}^{N}, X\right)$,

$$
f=(2 \pi)^{-N} \lim _{\substack{\varepsilon \rightarrow 0 \\ R \rightarrow \infty}} \int_{\varepsilon}^{R} \varphi_{t} * \psi_{t} * f \frac{d t}{t},
$$

where the limit is taken in $E\left(\mathbb{R}^{N}, X\right)$ for $E \in\left\{L_{p}, H_{1}\right.$, BMO : $\left.p \in(1, \infty)\right\}$. If we assume in addition that $\varphi, \psi \in L_{1}\left(\mathbb{R}^{N}\right)$, then the identity holds for all $f \in L_{p}\left(\mathbb{R}^{N}, X\right)$.

REMARK 4.5 (Characterization of homogeneous Sobolev spaces). Let $X$ be a UMD space and $p \in(1, \infty)$. Then, as noted above, Theorem $4.2(\mathrm{~b})$ 
implies that the space $L_{p}\left(\mathbb{R}^{N}, X\right)$ can be characterized in terms of the continuous wavelet transform. This is also true for the homogeneous Sobolev spaces

$$
\begin{aligned}
& \dot{H}_{p}^{s}\left(\mathbb{R}^{N}, X\right)=\left\{f \in \mathcal{S}^{\prime}\left(\mathbb{R}^{N}, X\right) / \mathcal{P}\left(\mathbb{R}^{N}, X\right):\|f\|_{\dot{H}_{p}^{s}\left(\mathbb{R}^{N}, X\right)}<\infty\right\} \\
& \|f\|_{\dot{H}_{p}^{s}\left(\mathbb{R}^{N}, X\right)}:=\left\|\mathcal{F}^{-1}\left(|\cdot|^{s} \widehat{f}\right)\right\|_{L_{p}\left(\mathbb{R}^{N}, X\right)},
\end{aligned}
$$

where $s$ is some real number and $\mathcal{P}\left(\mathbb{R}^{N}, X\right)$ are the polynomials on $\mathbb{R}^{N}$ with coefficients in $X$.

Indeed, assume that the wavelet $\psi \in L_{2}\left(\mathbb{R}^{N}\right)$ is chosen such that for all $\alpha \in\{0,1\}^{N}$ with $|\alpha| \leq l$, the distributional derivatives $D^{\alpha} \widehat{\psi}$ are represented by measurable functions,

$$
\sup _{|\xi|=1}\left(\int_{0}^{\infty} t^{2|\alpha|-2 s}\left|\left(D^{\alpha} \widehat{\psi}\right)(t \xi)\right|^{2} \frac{d t}{t}\right)^{1 / 2}<\infty,
$$

and

$$
\inf _{|\xi|=1}\left(\int_{0}^{\infty} t^{-2 s}|\widehat{\psi}(t \xi)|^{2} \frac{d t}{t}\right)^{1 / 2}>0 .
$$

If we denote by $\mathcal{H}_{s}$ the Hilbert space $L_{2}\left((0, \infty), t^{-2 s-1} d t\right)$, then

$$
\left\|\mathcal{W}_{\psi} f\right\|_{L_{p}\left(\mathbb{R}^{N}, \gamma\left(\mathcal{H}_{s}, X\right)\right)} \sim\|f\|_{\dot{H}_{p}^{s}\left(\mathbb{R}^{N}, X\right)} .
$$

This can be shown in the same way as Theorem 4.2: as multiplier functions take

$$
M_{\psi, s}: \mathbb{R}^{N} \backslash\{0\} \rightarrow \mathcal{L}\left(X, \gamma\left(\mathcal{H}_{s}, X\right)\right), \quad M_{\psi, s}(\xi) x=\left[t \mapsto|t \xi|^{-s} \widehat{\psi}(t \xi) x\right]
$$

and

$$
N_{\varphi, s}: \mathbb{R}^{N} \backslash\{0\} \rightarrow \mathcal{L}\left(\gamma\left(\mathcal{H}_{s}, X\right), X\right), \quad N_{\varphi, s}(\xi) y:=y\left(|\cdot \xi|^{-s} \widehat{\varphi}(\cdot \xi)\right),
$$

where $\varphi \in L_{2}\left(\mathbb{R}^{N}\right)$ is defined by

$$
\widehat{\varphi}(\xi):=\left(\int_{0}^{\infty}|t \xi|^{-2 s}|\widehat{\psi}(t \xi)|^{2} \frac{d t}{t}\right)^{-1} \overline{\widehat{\psi}(\xi)}, \quad \xi \in \mathbb{R}^{N} .
$$

REMARK 4.6. In Theorem 4.2, it is necessary that $X$ has the UMD property. Indeed, the UMD property can be characterized in terms of the wavelet transform: Let $X$ be an arbitrary Banach space and let $\Psi$ be the set of all wavelets in $L_{2}(\mathbb{R})$ satisfying $(\mathrm{C} 1)$ and $(\mathrm{C} 2)$ with $l=1$. Then the following three assertions are equivalent:

(i) $X$ is a UMD space.

(ii) (6) holds for all $\psi \in \Psi$.

(iii) There exists $\psi \in \Psi$ such that (6) holds for $\psi$ and $\widetilde{\psi}=\mathcal{F}^{-1}(\operatorname{sign} \cdot \widehat{\psi})$. 
That (i) implies (ii) has been shown in Theorem 4.2. (ii) $\Rightarrow$ (iii) is clear since $\mathcal{F}^{-1}(\operatorname{sign} \cdot \widehat{\psi}) \in \Psi$ if $\psi \in \Psi$. It remains to show that (iii) implies (i). For this we prove that the Hilbert transform $\mathcal{H}$ extends to a bounded linear operator on $L_{2}(\mathbb{R}, X)$. Let $f \in \mathcal{S}(\mathbb{R}, X)$. Then

$$
\mathcal{W}_{\psi} \mathcal{H} f=\mathcal{F}^{-1}(-i \pi \operatorname{sign}(\cdot) M(\cdot) \widehat{f}(\cdot))=-i \pi \mathcal{W}_{\widetilde{\psi}} f .
$$

Now we use $(6)$ for $\psi$ and $\widetilde{\psi}$.

Our approach to the vector-valued wavelet transform can be used to generalize the localization operators from $[9,21,39]$ to localization operators with operator-valued symbols on vector-valued $L_{p}$ spaces.

Let $F: \mathbb{R}_{*} \times \mathbb{R}^{N} \rightarrow \mathcal{L}(X, Z)$ be strongly measurable and bounded and let $\varphi, \psi \in L_{2}\left(\mathbb{R}^{N}\right)$ be wavelets satisfying $(\mathrm{C} 1)$. We would like to give meaning to the localization formula

$$
L_{F, \varphi, \psi} f:=\mathcal{M}_{\varphi}\left[F(\cdot, \cdot)\left(\mathcal{W}_{\psi} f\right)(\cdot, \cdot)\right], \quad f \in L_{p}\left(\mathbb{R}^{N}, X\right),
$$

and show that it defines a bounded operator from $L_{p}\left(\mathbb{R}^{N}, X\right)$ to $L_{p}\left(\mathbb{R}^{N}, Z\right)$. Here the name "localization operator" is motivated by the important example $F(\cdot, \cdot)=\chi_{G}(\cdot, \cdot) \operatorname{Id}_{X}$, where $G$ is a measurable subset of $R_{*} \times \mathbb{R}^{N}$ and $X=Z$. If $G=\mathbb{R}_{*} \times \mathbb{R}^{N}$ and $\varphi$ is chosen as in the proof of Theorem 4.2(b), then (10) corresponds to the reconstruction formula from Remark 4.4.

Now assume that

$$
\sup _{s \in \mathbb{R}^{N}} R\left(\left\{F(t, s): t \in \mathbb{R}_{*}\right\}\right)<\infty .
$$

But by [26, Sect. 5] we have for all $g \in \gamma\left(\mathbb{R}_{*}, X\right)$ the estimate

$$
\|F(\cdot, s) g(\cdot)\|_{\gamma\left(\mathbb{R}_{*}, Y\right)} \leq R\left(\left\{F(t, s): t \in \mathbb{R}_{*}\right\}\right)\|g\|_{\gamma\left(\mathbb{R}_{*}, X\right)} .
$$

Hence $(\widetilde{F} f)(t, s)=F(t, s)[f(t, s)]$ defines a bounded operator

$$
\widetilde{F}: L_{p}\left(\mathbb{R}^{N}, \gamma\left(\mathbb{R}_{*}, X\right)\right) \rightarrow L_{p}\left(\mathbb{R}^{N}, \gamma\left(\mathbb{R}_{*}, Z\right)\right) .
$$

Since $L_{F, \varphi, \psi}=\mathcal{M}_{\varphi} \circ \widetilde{F} \circ \mathcal{W}_{\psi}$, we have shown

Proposition 4.7. Let $X$ and $Z$ be UMD spaces, $p \in(1, \infty)$ and let $\varphi, \psi \in L_{2}\left(\mathbb{R}^{N}\right)$ be wavelets satisfying $(\mathrm{C} 1)$. If $F: \mathbb{R}_{*} \times \mathbb{R}^{N} \rightarrow \mathcal{L}(X, Z)$ is strongly measurable and satisfies (11), then the operator $L_{F, \varphi, \psi}$ is bounded from $L_{p}\left(\mathbb{R}^{N}, X\right)$ to $L_{p}\left(\mathbb{R}^{N}, Z\right)$.

REMARK 4.8. Note that (11) is satisfied if $F(\cdot, \cdot)=m(\cdot, \cdot) \operatorname{Id}_{X}$, where $m: \mathbb{R}_{*} \times \mathbb{R}^{N} \rightarrow \mathbb{C}$ is a bounded measurable function.

5. The semidiscrete wavelet transform. Let $X$ be a UMD space with Fourier type $r \in(1,2], N \in \mathbb{N}$ and $l:=[N / r]+1$. We now assume that $\psi \in L_{2}\left(\mathbb{R}^{N}\right)$ and $a>1$ with: 
(S1) for all $\alpha \in\{0,1\}^{N}$ with $|\alpha| \leq l$, the distributional derivatives $D^{\alpha} \widehat{\psi}$ are represented by measurable functions and

$$
\begin{array}{r}
C_{\alpha}^{\prime}(\psi):=\sup _{1 \leq|\xi|<a}\left(\sum_{j \in \mathbb{Z}} a^{2 j|\alpha|}\left|\left(D^{\alpha} \widehat{\psi}\right)\left(a^{j} \xi\right)\right|^{2}\right)^{1 / 2}<\infty, \\
\text { (S2) } c_{0}^{\prime}(\psi):=\inf _{1 \leq|\xi|<a}\left(\sum_{j \in \mathbb{Z}}\left|\widehat{\psi}\left(a^{j} \xi\right)\right|^{2}\right)^{1 / 2}>0 .
\end{array}
$$

REMARK 5.1. It is not hard to see that (S1) implies (C1), and (S2) implies (C2). Indeed, suppose (S1) holds. Let $\omega \in \mathbb{R}^{N}$ with $|\omega|=1$ and write $\xi=t \omega$. Integrating over $1 \leq t \leq a$ with respect to the measure $\frac{d t}{t}$ yields

$$
\int_{1}^{a} \sum_{j \in \mathbb{Z}} a^{2 j|\alpha|}\left|\left(D^{\alpha} \widehat{\psi}\right)\left(a^{j} t \omega\right)\right|^{2} \frac{d t}{t} \leq C_{\alpha}^{\prime}(\psi)^{2} \log a .
$$

But

$$
\begin{aligned}
& \int_{1}^{a} \sum_{j \in \mathbb{Z}} a^{2 j|\alpha|}\left|\left(D^{\alpha} \widehat{\psi}\right)\left(a^{j} t \omega\right)\right|^{2} \frac{d t}{t}=\sum_{j \in \mathbb{Z}} \int_{a^{j}}^{a^{j+1}} a^{2 j|\alpha|}\left|\left(D^{\alpha} \widehat{\psi}\right)(t \omega)\right|^{2} \frac{d t}{t} \\
& \geq \sum_{j \in \mathbb{Z}} \int_{a^{j}}^{a^{j+1}}\left(\frac{t}{a}\right)^{2|\alpha|}\left|\left(D^{\alpha} \widehat{\psi}\right)(t \omega)\right|^{2} \frac{d t}{t}=a^{-2|\alpha|} \int_{0}^{\infty} t^{2|\alpha|}\left|\left(D^{\alpha} \widehat{\psi}\right)(t \omega)\right|^{2} \frac{d t}{t} .
\end{aligned}
$$

Therefore (C1) holds. For (S2) we proceed in a similar way (see also [7]).

Definition 5.2. Let $a>0$. The semidiscrete wavelet transform $\mathcal{W}_{a, \psi} f$ of a function $f \in \mathcal{S}\left(\mathbb{R}^{N}, X\right)$ with respect to the wavelet $\psi$ is given by

$$
\left(\mathcal{W}_{a, \psi} f\right)(j, s):=\left(\mathcal{W}_{\psi} f\right)\left(a^{j}, s\right), \quad j \in \mathbb{Z}, s \in \mathbb{R}^{N} .
$$

TheOREM 5.3. Let $X$ be a UMD space with Fourier type $r \in(1,2], n \in \mathbb{N}$ and $l:=[N / r]+1$.

(a) If $\psi \in L_{2}\left(\mathbb{R}^{N}\right)$ and $a>1$ with $(\mathrm{S} 1)$, then $\mathcal{W}_{a, \psi}$ defines a bounded linear operator from $E\left(\mathbb{R}^{N}, X\right)$ to $E\left(\mathbb{R}^{N}, \gamma(\mathbb{Z}, X)\right)$, where $E \in\left\{L_{p}, H_{1}, \mathrm{BMO}\right\}$.

(b) If $\psi \in L_{2}\left(\mathbb{R}^{N}\right)$ and $a>1$ satisfy (S1) and (S2), then

$$
\left\|\mathcal{W}_{a, \psi} f\right\|_{E\left(\mathbb{R}^{N}, \gamma(\mathbb{Z}, X)\right)} \sim\|f\|_{E\left(\mathbb{R}^{N}, X\right)}
$$

for all $f \in E\left(\mathbb{R}^{N}, X\right)$ with $E \in\left\{L_{p}, H_{1}, \mathrm{BMO}\right\}$.

The proof of this theorem is very similar to the one in the continuous case and will be omitted here.

REMARK 5.4 (Classical square functions). (a) If $X=L_{q}(\Omega, \mu)$ for some $q \in(1, \infty)$ and a $\sigma$-finite measure space $(\Omega, \Sigma, \mu)$, then the norm equivalence 
in Theorem 5.3 reads (cf. Remark 2.18)

$$
\left\|\left(\sum_{k \in \mathbb{Z}}\left|\psi_{a^{j}} * f\right|^{2}\right)^{1 / 2}\right\|_{L_{p}\left(\mathbb{R}^{N}, X\right)} \sim\|f\|_{L_{p}\left(\mathbb{R}^{N}, X\right)} .
$$

(b) We can also formulate an analogue of Remark 4.5 for the semidiscrete wavelet transform. This gives a vector-valued version of the fact that for $p \in(1, \infty)$ and $s \in \mathbb{R}$ the homogeneous Triebel-Lizorkin spaces $\dot{F}_{p, 2}^{s}\left(\mathbb{R}^{N}\right)$ are isomorphic to the homogeneous Sobolev spaces $\dot{H}_{p}^{s}\left(\mathbb{R}^{N}\right)$ (cf. [35, pp. $168 \mathrm{ff}$.], $[16])$.

REMARK 5.5 (Reconstruction formula). Suppose that $\psi, \varphi \in L_{2}\left(\mathbb{R}^{N}\right)$ and $a>1$ satisfy (S1) with

$$
\sum_{j \in \mathbb{Z}} \widehat{\varphi}\left(a^{j} \xi\right) \widehat{\psi}\left(a^{j} \xi\right)=1
$$

for almost all $\xi \in \mathbb{R}^{N} \backslash\{0\}$. As in Remark 4.4 we can show that

$$
f=\mathcal{M}_{a, \varphi} \mathcal{W}_{a, \psi} f
$$

for all $f \in E\left(\mathbb{R}^{N}, X\right)$. If $f \in \mathcal{S}\left(\mathbb{R}^{N}, X\right) \cap E\left(\mathbb{R}^{N}, X\right)$, this reconstruction formula can be written as

$$
f=(2 \pi)^{-N} \sum_{j \in \mathbb{Z}} \varphi_{a^{j}} * \psi_{a^{j}} * f .
$$

If we assume in addition that $\varphi, \psi \in L_{1}\left(\mathbb{R}^{N}\right)$, then the identity holds for all $L_{p}\left(\mathbb{R}^{N}, X\right)$.

REMARK 5.6 (Localization operators). Let $X$ and $Y$ be UMD spaces and let $F: \mathbb{Z} \times \mathbb{R}^{N} \rightarrow \mathcal{L}(X, Y)$ be a strongly measurable function satisfying the R-boundedness assumption

$$
\sup _{s \in \mathbb{R}^{N}} R(\{F(j, s): j \in \mathbb{Z}\})<\infty .
$$

In the same way as in (10) we can define the localization operator $L_{F, \varphi, \psi}$ and show that it is bounded from $L_{p}\left(\mathbb{R}^{N}, X\right)$ to $L_{p}\left(\mathbb{R}^{N}, Y\right)$.

If we take $X=Y, F(j, \cdot) \equiv \varepsilon_{j}$ with $\varepsilon_{j} \in\{-1,1\}$ and $\varphi, \psi$ are as in Remark 5.5, then we deduce that the reconstruction formula (13) converges unconditionally in $L_{p}\left(\mathbb{R}^{N}, X\right)$.

6. The discrete wavelet transform. Let $a>1, b>0$. For $\psi \in$ $L_{2}\left(\mathbb{R}^{N}\right)$ and $j \in \mathbb{Z}, k \in \mathbb{Z}^{N}$ we write

$$
\psi_{j, k}^{(a, b)}(u)=a^{-N j / 2} \psi\left(a^{-j} u-b k\right) .
$$


We will consider the following two conditions on $\psi$ :

(D1) $\left\{\psi_{j, k}^{(a, b)}\right\}$ is a frame, i.e. for all $f \in L_{2}\left(\mathbb{R}^{N}\right)$,

$$
A\|f\|_{2}^{2} \leq \sum_{j \in \mathbb{Z}} \sum_{k \in \mathbb{Z}^{N}}\left|\left\langle\psi_{j, k}^{(a, b)}, f\right\rangle\right|^{2} \leq B\|f\|_{2}^{2} .
$$

(D2) $\psi$ satisfies the estimates

$$
|\psi(u)|,|\nabla \psi(u)| \leq c(1+|u|)^{-(N+1)-\varepsilon} .
$$

REMARK 6.1. If $\psi \in L_{2}\left(\mathbb{R}^{N}\right)$ satisfies (D1), then for almost all $\xi \in \mathbb{R}$,

$$
A \leq\left(\frac{2 \pi}{b}\right)^{N} \sum_{j \in \mathbb{Z}}\left|\widehat{\psi}\left(a^{j} \xi\right)\right|^{2} \leq B .
$$

This can be shown in a similar way to $[9$, pp. 63-66] (see also [7]).

If in addition (D2) holds, then $\psi \in L_{1}\left(\mathbb{R}^{N}\right)$. Hence $\widehat{\psi}$ is continuous and therefore $\int_{\mathbb{R}^{N}} \psi(u) d u=\widehat{\psi}(0)=0$.

In analogy to Section 5, where we studied localization operators for the continuous wavelet transform, we now investigate localization (or filtering) operators for the discrete wavelet transform. Again the multiplier function is operator-valued. Our next result can be seen as an extension of a martingale transform theorem from [19].

Theorem 6.2. Let $X, Y$ be UMD spaces, $p \in(1, \infty)$, and $\tau=\left\{M_{j, k}\right.$ : $\left.j \in \mathbb{Z}, k \in \mathbb{Z}^{N}\right\}$ an $R$-bounded subset of $\mathcal{L}(X, Y)$. If $\psi, \varphi \in L_{2}\left(\mathbb{R}^{N}\right)$ satisfy (D1) and (D2), then the operator

$$
L f=\sum_{j \in \mathbb{Z}} \sum_{k \in \mathbb{Z}^{N}} M_{j, k}\left\langle\psi_{j, k}^{(a, b)}, f\right\rangle \varphi_{j, k}^{(a, b)}, \quad f \in X \otimes \mathcal{S}\left(\mathbb{R}^{N}\right),
$$

extends to a bounded linear operator from $E\left(\mathbb{R}^{N}, X\right)$ to $E\left(\mathbb{R}^{N}, Y\right)$, where $E \in\left\{L_{p}, H_{1}\right\}$. The sum in (14) converges unconditionally in $E\left(\mathbb{R}^{N}, X\right)$ for all $f \in E\left(\mathbb{R}^{N}, X\right)$.

As a corollary we obtain a reconstruction formula for the discrete wavelet transform. For this we will need an additional assumption on the wavelets $\varphi, \psi$ :

(D3) for all $f \in \mathcal{S}\left(\mathbb{R}^{N}\right)$,

$$
f=\sum_{j \in \mathbb{Z}} \sum_{k \in \mathbb{Z}^{N}}\left\langle\psi_{j, k}^{(a, b)}, f\right\rangle \varphi_{j, k}^{(a, b)} .
$$

Corollary 6.3. Let $X$ be a UMD space, $p \in(1, \infty)$, and $f \in E\left(\mathbb{R}^{N}, X\right)$, where $E \in\left\{L_{p}, H_{1}\right\}$. If $\psi, \varphi \in L_{2}\left(\mathbb{R}^{N}\right)$ satisfy (D1) and (D2), then the series

$$
\sum_{j \in \mathbb{Z}} \sum_{k \in \mathbb{Z}^{N}}\left\langle\psi_{j, k}^{(a, b)}, f\right\rangle \varphi_{j, k}^{(a, b)}
$$


converges unconditionally in $E\left(\mathbb{R}^{N}, X\right)$. If, in addition, (D3) holds, then

$$
f=\sum_{j \in \mathbb{Z}} \sum_{k \in \mathbb{Z}^{N}}\left\langle\psi_{j, k}^{(a, b)}, f\right\rangle \varphi_{j, k}^{(a, b)}
$$

in $E\left(\mathbb{R}^{N}, X\right)$.

This corollary is a generalization of a theorem by Figiel [13]. See also [23] for a related result on vector-valued Hardy spaces.

Before we come to the proof of Theorem 6.2, we give some examples of wavelets $\varphi, \psi$ for which the assumptions (D1)-(D3) are satisfied.

EXAMPLE 6.4 .

(a) Suppose $\left\{\psi_{j, k}^{(a, b)}\right\}$ is an orthonormal basis in $L_{2}\left(\mathbb{R}^{N}\right)$ and $\varphi=\psi$. Then (D1) and (D3) hold. For the existence of functions $\psi$ such that in addition (D2) is satisfied, see e.g. [9], [29].

(b) Suppose $\left\{\psi_{j, k}^{(a, b)}\right\}$ is a tight frame, i.e., $A=B$ in (D1), and $\varphi=\psi$. Then (D3) holds (cf. [9], [29]).

(c) Suppose $\psi \in \mathcal{S}\left(\mathbb{R}^{N}\right)$ with $\operatorname{supp} \widehat{\psi} \subseteq\left\{\xi \in \mathbb{R}^{N}: 1 / 2 \leq|\xi| \leq 2\right\}$ and $|\widehat{\psi}(\xi)| \geq c>0$ for $3 / 5 \leq|\xi| \leq 5 / 3$. Then conditions (D1), (D2) are satisfied with $a=2$ (cf. [9], [29]). Moreover, by [16, pp. 54-56], there is a $\varphi \in L_{2}\left(\mathbb{R}^{N}\right)$ satisfying (D1) and (D2) such that (D3) holds for $\varphi, \psi$.

For the proof of Theorem 6.2, we need a vector-valued version of the classical $T(1)$ theorem. For scalar-valued kernels, such a theorem was proved by Figiel [14]. Since our kernels will be operator-valued, we use the following result, which was proved in a much more general version in [24].

To state this theorem we need the following notion. We say that $\varphi$ is a normalized bump function associated with the unit ball if $\varphi \in C_{\mathrm{c}}^{\infty}\left(\mathbb{R}^{N}\right)$ with $\operatorname{supp} \varphi \subseteq \bar{B}(0,1)$ and $\left\|D^{\alpha} \varphi\right\|_{\infty} \leq 1$ for all $|\alpha| \leq n$, where $n$ is a large fixed number. Hence the $L_{2}\left(\mathbb{R}^{N}\right)$-norm of any normalized bump function is bounded by a constant only depending on $N$.

TheORem 6.5 ([24]). Let $X, Y$ be $U M D$ spaces, $p \in(1, \infty)$, and $\mathcal{K} \subseteq$ $L_{1}\left(\mathbb{R}^{N} \times \mathbb{R}^{N}, \mathcal{L}(X, Y)\right)$. For $K \in \mathcal{K}$ we define $T_{K}: L_{\infty}\left(\mathbb{R}^{N}, X\right) \rightarrow L_{1}\left(\mathbb{R}^{N}, Y\right)$ by

$$
T_{K} f=\int_{\mathbb{R}^{N}} K(\cdot, v) f(v) d v
$$

and assume that there is a constant $C<\infty$ such that, for all $K \in \mathcal{K}$,

(1) $K$ satisfies the standard $R$-estimates

$$
R\left(|u-v|^{N} K(u, v): u, v \in \mathbb{R}^{N}\right) \leq C,
$$




$$
\begin{aligned}
& R\left(|u-v|^{N+1} \frac{K(u, v)-K(u, \widetilde{v})}{|v-\widetilde{v}|}:|u-v|>2|v-\widetilde{v}|>0\right) \leq C, \\
& R\left(|u-v|^{N+1} \frac{K(u, v)-K(\widetilde{u}, v)}{|u-\widetilde{u}|}:|u-v|>2|u-\widetilde{u}|>0\right) \leq C,
\end{aligned}
$$

(2) $T_{K}(1)=T_{K}^{\prime}(1)=0$, i.e. $\int_{\mathbb{R}^{N}} K(\cdot, v) d v=\int_{\mathbb{R}^{N}} K(u, \cdot)^{\prime} d u=0$, and

(3) $T_{K}$ has the weak $R$-boundedness property, i.e. for every pair of normalized bump functions $\varphi, \widetilde{\varphi}$ associated with the unit ball we have

$$
R\left(\left\langle\varphi, T^{r, s} \widetilde{\varphi}\right\rangle: s>0, r \in \mathbb{R}^{N}\right) \leq C,
$$

where $\left\langle\varphi, T^{r, s} \widetilde{\varphi}\right\rangle \in \mathcal{L}(X, Y)$ is defined by

$$
\left\langle\varphi, T^{r, s} \widetilde{\varphi}\right\rangle x=r^{-N} \int_{\mathbb{R}^{N}} \varphi\left(\frac{t-s}{r}\right) T\left[\widetilde{\varphi}\left(\frac{\cdot-s}{r}\right) x\right](t) d t, \quad x \in X .
$$

Then each $T_{K}$ defines a bounded operator from $E\left(\mathbb{R}^{N}, X\right)$ to $E\left(\mathbb{R}^{N}, Y\right)$, where $E \in\left\{L_{p}, H_{1}\right\}$. The family $\left\{T_{K}: K \in \mathcal{K}\right\}$ is uniformly bounded in $\mathcal{L}\left(E\left(\mathbb{R}^{N}, X\right), E\left(\mathbb{R}^{N}, Y\right)\right)$.

Now we can prove Theorem 6.2.

Proof of Theorem 6.2. Let $E \in\left\{L_{p}, H_{1}: p \in(1, \infty)\right\}$.

SteP 1. Let $f \in E\left(\mathbb{R}^{N}, X\right)$. Since $E\left(\mathbb{R}^{N}, Y\right)$ has finite cotype, it does not contain $c_{0}$. Therefore the series $\sum_{j \in \mathbb{Z}} \sum_{k \in \mathbb{Z}^{N}} M_{j, k}\left\langle\psi_{j, k}^{(a, b)}, f\right\rangle \varphi_{j, k}^{(a, b)}$ converges unconditionally in $E\left(\mathbb{R}^{N}, Y\right)$ if and only if it converges weakly unconditionally in $E\left(\mathbb{R}^{N}, Y\right)$ (see e.g. [11, p. 45]). We will prove that there is a constant $C$ such that, for all finite subsets $F$ of $\mathbb{Z} \times \mathbb{Z}^{N}$, all $f \in E\left(\mathbb{R}^{N}, X\right)$ and all $g \in\left[E\left(\mathbb{R}^{N}, Y\right)\right]^{\prime}$,

$$
\left|\left\langle\sum_{(j, k) \in F} M_{j, k}\left\langle\psi_{j, k}^{(a, b)}, f\right\rangle \varphi_{j, k}^{(a, b)}, g\right\rangle_{E\left(\mathbb{R}^{N}, X\right)}\right| \leq C\|f\|_{E\left(\mathbb{R}^{N}, X\right)}\|g\|_{\left[E\left(\mathbb{R}^{N}, X\right)\right]^{\prime}} .
$$

STEP 2. For a finite subset $F$ of $\mathbb{Z} \times \mathbb{Z}^{N}$, we define an operator-valued kernel $K_{F}: \mathbb{R}^{N} \times \mathbb{R}^{N} \rightarrow \mathcal{L}(X, Y)$ by

$$
K_{F}(u, v)=\sum_{(j, k) \in F} M_{j, k} \psi_{j, k}^{(a, b)}(u) \varphi_{j, k}^{(a, b)}(v), \quad u, v \in \mathbb{R}^{N} .
$$

Then $K_{F} \in L_{1}\left(\mathbb{R}^{N} \times \mathbb{R}^{N}, \mathcal{L}(X, Y)\right)$ by (D2), and

$$
L_{F} f:=T_{K_{F}} f=\int_{\mathbb{R}^{N}} K_{F}(\cdot, v) f(v) d v, \quad f \in L_{\infty}\left(\mathbb{R}^{N}, X\right),
$$

is well-defined. We show that $\mathcal{K}=\left\{K_{F}: F\right.$ is a finite subset of $\left.\mathbb{Z} \times \mathbb{Z}^{N}\right\}$ satisfies the conditions of Theorem 6.5. 
STEP 3. To prove condition (1) of Theorem 6.5, we observe that

$$
|u-v|^{N} K_{F}(u, v)=|u-v|^{N} \sum_{(j, k) \in F} \psi_{j, k}^{(a, b)}(u) \varphi_{j, k}^{(a, b)}(v) M_{j, k} .
$$

By (D2) and Lemma 6.6 below, we see that

$$
\begin{aligned}
& \sup \left\{|u-v|^{N} \sum_{(j, k) \in F}\left|\psi_{j, k}^{(a, b)}(u) \varphi_{j, k}^{(a, b)}(v)\right|: u, v \in \mathbb{R}^{N}\right\} \\
& \leq \sup \left\{|u-v|^{N} \sum_{j \in \mathbb{Z}} \sum_{k \in \mathbb{Z}^{N}} a^{-N j}\right. \\
& \left.\quad \times\left[\left(1+\left|a^{-j} u-b k\right|\right)\left(1+\left|a^{-j} v-b k\right|\right)\right]^{-N-1-\varepsilon}: u, v \in \mathbb{R}^{N}\right\}=: C_{1}<\infty .
\end{aligned}
$$

Hence $\left\{|u-v|^{N} K_{F}(u, v): u, v \in \mathbb{R}^{N}\right\} \subseteq C_{1} \operatorname{absco}(\tau)$ and therefore

$$
R\left(|u-v|^{N} K_{F}(u, v): u, v \in \mathbb{R}^{N}\right) \leq 2 C_{1} R(\tau) .
$$

Next we consider

$$
\begin{aligned}
&|u-v|^{N+1} \frac{K_{F}(u, v)-K_{F}(u, \widetilde{v})}{|v-\widetilde{v}|} \\
&=|u-v|^{N+1} \sum_{(j, k) \in F} \psi_{j, k}^{(a, b)}(u) \frac{\varphi_{j, k}^{(a, b)}(v)-\varphi_{j, k}^{(a, b)}(\widetilde{v})}{|v-\widetilde{v}|} M_{j, k} .
\end{aligned}
$$

But

$$
\begin{aligned}
& \sup \left\{|u-v|^{N+1} \sum_{(j, k) \in F}\left|\psi_{j, k}^{(a, b)}(u)\right|\right. \\
& \left.\quad \times \frac{\left|\varphi_{j, k}^{(a, b)}(v)-\varphi_{j, k}^{(a, b)}(\widetilde{v})\right|}{|v-\widetilde{v}|}:|u-v|>2|v-\widetilde{v}|>0\right\} \\
& \leq c_{N} \sup \left\{|u-w|^{N+1} \sum_{(j, k) \in F}\left|\psi_{j, k}^{(a, b)}(u)\right|\left|\left(\nabla \varphi_{j, k}^{(a, b)}\right)(w)\right|: u, w \in \mathbb{R}^{N}\right\} \\
& \leq c_{N} \sup \left\{|u-w|^{N+1} \sum_{j \in \mathbb{Z}} \sum_{k \in \mathbb{Z}^{N}} a^{-(N+1) j}\right. \\
& \left.\quad \times\left[\left(1+\left|a^{-j} u-b k\right|\right)\left(1+\left|a^{-j} w-b k\right|\right)\right]^{-N-1-\varepsilon}: u, w \in \mathbb{R}^{N}\right\}=: C_{2}
\end{aligned}
$$

by (D2) and Lemma 6.6 below. So

$$
R\left(|u-v|^{N+1} \frac{K_{F}(u, v)-K_{F}(u, \widetilde{v})}{|v-\widetilde{v}|}:|u-v|>2|v-\widetilde{v}|>0\right) \leq 2 C_{2} R(\tau) .
$$

In the same way we show

$$
R\left(|u-v|^{N+1} \frac{K_{F}(u, v)-K_{F}(u, \widetilde{v})}{|u-\widetilde{u}|}:|u-v|>2|u-\widetilde{u}|>0\right) \leq 2 C_{3} R(\tau) .
$$


SteP 4. Condition (2) of Theorem 6.5 follows easily from the fact that $\int_{\mathbb{R}^{N}} \psi(t) d t=\int_{\mathbb{R}^{N}} \varphi(t) d t=0$. Indeed,

$$
\begin{aligned}
\int_{\mathbb{R}^{N}} K_{F}(\cdot, v) d v & =\int_{\mathbb{R}^{N}} \sum_{(j, k) \in F} M_{j, k} \psi_{j, k}^{(a, b)}(\cdot) \varphi_{j, k}^{(a, b)}(v) d v \\
& =\sum_{(j, k) \in F} M_{j, k} \psi_{j, k}^{(a, b)}(\cdot) \int_{\mathbb{R}^{N}} \varphi_{j, k}^{(a, b)}(v) d v=0
\end{aligned}
$$

and

$$
\begin{aligned}
\int_{\mathbb{R}^{N}} K_{F}(u, \cdot)^{\prime} d u & =\int_{\mathbb{R}^{N}} \sum_{(j, k) \in F} M_{j, k}^{\prime} \psi_{j, k}^{(a, b)}(u) \varphi_{j, k}^{(a, b)}(\cdot) d u \\
& =\sum_{(j, k) \in F} M_{j, k}^{\prime} \varphi_{j, k}^{(a, b)}(\cdot) \int_{\mathbb{R}^{N}} \psi_{j, k}^{(a, b)}(u) d u=0 .
\end{aligned}
$$

SteP 5. To prove (3), let $\varphi, \widetilde{\varphi}$ be a pair of normalized bump functions associated with the unit ball. We obtain, for $r>0$ and $s \in \mathbb{R}^{N}$,

$$
\begin{aligned}
\left\langle\varphi, L_{F}^{r, s} \widetilde{\varphi}\right\rangle & =r^{-N}\left\langle\varphi\left(\frac{\cdot-s}{r}\right), \sum_{(j, k) \in F} M_{j, k}\left\langle\psi_{j, k}^{(a, b)}, \widetilde{\varphi}\left(\frac{\cdot-s}{r}\right)\right\rangle \varphi_{j, k}^{(a, b)}\right\rangle \\
& =r^{-N} \sum_{(j, k) \in F}\left\langle\psi_{j, k}^{(a, b)}, \widetilde{\varphi}\left(\frac{\cdot-s}{r}\right)\right\rangle\left\langle\varphi\left(\frac{\cdot-s}{r}\right), \varphi_{j, k}^{(a, b)}\right\rangle M_{j, k}
\end{aligned}
$$

with

$$
\begin{aligned}
& r^{-N} \sum_{(j, k) \in F}\left|\left\langle\psi_{j, k}^{(a, b)}, \widetilde{\varphi}\left(\frac{\cdot-s}{r}\right)\right\rangle\left\langle\varphi\left(\frac{\cdot-s}{r}\right), \varphi_{j, k}^{(a, b)}\right\rangle\right| \\
& \quad \leq r^{-N}\left(\sum_{(j, k) \in F}\left|\left\langle\psi_{j, k}, \widetilde{\varphi}\left(\frac{\cdot-s}{r}\right)\right\rangle\right|^{2}\right)^{1 / 2}\left(\sum_{(j, k) \in F}\left|\left\langle\varphi\left(\frac{\cdot-s}{r}\right), \varphi_{j, k}\right\rangle\right|^{2}\right)^{1 / 2} \\
& \quad \leq B r^{-N}\left\|\varphi\left(\frac{\cdot-s}{r}\right)\right\|_{2}\left\|\widetilde{\varphi}\left(\frac{\cdot-s}{r}\right)\right\|_{2}=B\|\varphi\|_{2}\|\widetilde{\varphi}\|_{2}
\end{aligned}
$$

where $B$ is the constant from assumption (D1). Since $\|\varphi\|_{2},\|\widetilde{\varphi}\|_{2}$ are bounded by some constant only depending on $N$, there is a constant $C_{4}$ such that $\left\{\left\langle\varphi, L_{F}^{r, s} \widetilde{\varphi}\right\rangle: r>0, s \in \mathbb{R}^{N}\right\} \subseteq C_{4} \operatorname{absco}(\tau)$. Hence

$$
R\left(\left\langle\varphi, L_{F}^{a, b} \widetilde{\varphi}\right\rangle: a>0, b \in \mathbb{R}^{N}\right) \leq 2 C_{4} R(\tau) .
$$

Step 6. Now, by Steps 2 to 5, we can apply Theorem 6.5 and deduce that the set

$$
\left\{L_{F}: F \text { is a finite subset of } \mathbb{Z} \times \mathbb{Z}^{N}\right\}
$$

is bounded in $\mathcal{L}\left(E\left(\mathbb{R}^{N}, X\right), E\left(\mathbb{R}^{N}, Y\right)\right)$. Step 1 now implies that the sum in (14) converges unconditionally in $E\left(\mathbb{R}^{N}, Y\right)$ for all $f \in E\left(\mathbb{R}^{N}, X\right)$. By the 
uniform boundedness principle, $L$ defines an bounded linear operator from $E\left(\mathbb{R}^{N}, X\right)$ to $E\left(\mathbb{R}^{N}, Y\right)$.

The following technical lemma was crucial in the proof of Theorem 6.2. The proof is standard (cf. e.g. [30], [9]) and is omitted here.

Lemma 6.6. Let $\gamma>1$ and $N \in \mathbb{N}$. Then there is a constant $C$, only depending on $\gamma, a, b$, and $N$, such that for all $x, y \in \mathbb{R}^{N}$ with $x \neq y$,

$$
\sum_{j \in \mathbb{Z}} \sum_{k \in \mathbb{Z}^{N}} a^{-N j}\left[\left(1+\left|a^{-j} x-b k\right|\right)\left(1+\left|a^{-j} y-b k\right|\right)\right]^{-N \gamma} \leq C|x-y|^{-N} .
$$

REMARK 6.7. In the multi-dimensional case, one is also interested in orthonormal wavelet bases generated by shifts and dilates of a finite collection $\Psi$ of $L_{2}$-functions. (A prominent example is the tensor wavelets generated by tensor products from a one-dimensional wavelet and its corresponding scaling function, see e.g. [9, pp. 313-319].) If we assume that in addition each $\psi \in \Psi$ satisfies condition (D2) and that the parameters $a, b$ are the same for all $\psi \in \Psi$, then our methods can be applied to obtain results corresponding to Theorem 6.2 and Corollary 6.3 in this setting.

Acknowledgements. The first author wants to thank the Alexander von Humboldt Foundation for their support which made it possible to spend a wonderful year at the University of South Carolina. She also wants to thank M. Girardi, A. Schep and the whole Department of Mathematics at USC for their kind hospitality. Finally, she wants to thank J. van Neerven and B. Haak for inspiring discussions on Lemma 3.1.

The second author wants to acknowledge the support of the Volkswagen Stiftung for his sabbatical semester at the University of South Carolina and the support of the DFG through grant WE 2847/1-1.

\section{References}

[1] D. J. Aldous, Unconditional bases and martingales in $L_{p}(F)$, Math. Proc. Cambridge Philos. Soc. 85 (1979), 117-123.

[2] H. Amann, Linear and Quasilinear Parabolic Problems. Vol. I, Abstract Linear Theory, Monogr. Math. 89, Birkhäuser Boston, Boston, MA, 1995.

[3] J. Bergh and J. Löfström, Interpolation Spaces. An Introduction, Grundlehren Math. Wiss. 223, Springer, Berlin, 1976.

[4] J. Bourgain, A Hausdorff-Young inequality for B-convex Banach spaces, Pacific J. Math. 101 (1982), 255-262.

[5] - Vector-valued Hausdorff-Young inequalities and applications, in: Geometric Aspects of Functional Analysis (1986/87), Lecture Notes in Math. 1317, Springer, Berlin, 1988, 239-249.

[6] - Vector-valued singular integrals and the $H^{1}-B M O$ duality, in: Probability Theory and Harmonic Analysis (Cleveland, OH, 1983), Monogr. Textbooks Pure Appl. Math. 98, Dekker, New York, 1986, 1-19. 
[7] C. K. Chui and X. L. Shi, Inequalities of Littlewood-Paley type for frames and wavelets, SIAM J. Math. Anal. 24 (1993), 263-277.

[8] D. L. Cohn, Measure Theory, Birkhäuser Boston, Boston, MA, 1980.

[9] I. Daubechies, Ten Lectures on Wavelets, CBMS-NSF Reg. Conf. Ser. Appl. Math. 61, SIAM, Philadelphia, PA, 1992.

[10] J. Diestel, H. Jarchow and A. Tonge, Absolutely Summing Operators, Cambridge Stud. Adv. Math. 43, Cambridge Univ. Press, Cambridge, 1995.

[11] J. Diestel, Sequences and Series in Banach Spaces, Grad. Texts in Math. 92, Springer, New York, 1984.

[12] P. Enflo, Banach spaces which can be given an equivalent uniformly convex norm, Israel J. Math. 13 (1972), 281-288.

[13] T. Figiel, On equivalence of some bases to the Haar system in spaces of vector-valued functions, Bull. Polish Acad. Sci. Math. 36 (1988), 119-131.

[14] —, Singular integral operators: a martingale approach, in: Geometry of Banach Spaces (Strobl, 1989), London Math. Soc. Lecture Note Ser. 158, Cambridge Univ. Press, Cambridge, 1990, 95-110.

[15] T. Figiel and N. Tomczak-Jaegermann, Projections onto Hilbertian subspaces of Banach spaces, Israel J. Math. 33 (1979), 155-171.

[16] M. Frazier, B. Jawerth and G. Weiss, Littlewood-Paley Theory and the Study of Function Spaces, CBMS Reg. Conf. Ser. Math. 79, Amer. Math. Soc., Providence, RI, 1991.

[17] M. Girardi and L. Weis, Operator-valued Fourier multiplier theorems on Besov spaces, Math. Nachr. 251 (2003), 34-51.

[18] —, - Operator-valued Fourier multiplier theorems on $L_{p}(X)$ and geometry of Banach spaces, J. Funct. Anal. 204 (2003), 320-354.

[19] —, - Operator-valued martingale transforms and R-boundedness, Illinois J. Math. 49 (2005), 487-516.

[20] B. Haak, Kontrolltheorie in Banachräumen und quadratische Abschätzungen, Dissertation, Universitätsverlag Karlsruhe, 2004.

[21] M. Holschneider, Wavelets. An Analysis Tool, Oxford Math. Monogr., Clarendon Press, New York, 1995.

[22] T. Hytönen, Fourier embeddings and Mihlin-type multiplier theorems, Math. Nachr. 274/275 (2004), 74-103.

[23] - Vector-valued wavelets and the Hardy space $H^{1}\left(\mathbb{R}^{n}, X\right)$, Studia Math. 172 (2006), 125-147.

[24] T. Hytönen and L. Weis, A T1 theorem for integral transformations with operatorvalued kernel, J. Reine Angew. Math. 599 (2006), 155-200.

[25] C. Kaiser, Wavelet transforms for functions with values in Lebesgue spaces, in: Proc. SPIE Optics and Photonics 2005 Conf. on Mathematical Methods: Wavelets XI 5914, 2005.

[26] N. Kalton and L. Weis, The $H^{\infty}$-functional calculus and square function estimates, preprint.

[27] S. Kwapien, Isomorphic characterizations of inner product spaces by orthogonal series with vector valued coefficients, Studia Math. 44 (1972), 583-595.

[28] V. Linde, V. I. Tarieladze and S. A. Chobanyan, Characterization of certain classes of Banach spaces by properties of Gaussian measures, Teor. Veroyatnost. i Primenen. 25 (1980), no. 1, 162-167 (in Russian); English transl.: Theory Probab. Appl. 25 (1980), no. 1, 159-164.

[29] A. K. Louis, P. Maaß und A. Rieder, Wavelets, 2nd ed., Teubner Studienbücher Math., Teubner, Stuttgart, 1998 (in German). 
[30] Y. Meyer and R. Coifman, Wavelets, Calderón-Zygmund and Multilinear Operators, Cambridge Stud. Adv. Math. 48, Cambridge Univ. Press, Cambridge, 1997.

[31] J. Peetre, Sur la transformation de Fourier des fonctions à valeurs vectorielles, Rend. Sem. Mat. Univ. Padova 42 (1969), 15-26.

[32] G. Pisier, The Volume of Convex Bodies and Banach Space Geometry, Cambridge Tracts in Math. 94, Cambridge Univ. Press, Cambridge, 1989.

[33] E. M. Stein, Harmonic Analysis: Real-Variable Methods, Orthogonality, and Oscillatory Integrals, Princeton Math. Ser. 43, Princeton Univ. Press, Princeton, NJ, 1993.

[34] N. Tomczak-Jaegermann, Banach-Mazur Distances and Finite-Dimensional Operator Ideals, Pitman Monogr. Surveys Pure Appl. Math. 38, Longman Sci. \& Tech., Harlow, 1989.

[35] H. Triebel, Interpolation Theory, Function Spaces, Differential Operators, NorthHolland Math. Library 18, North-Holland, Amsterdam, 1978.

[36] —, Theory of Function Spaces. II, Monogr. Math. 84, Birkhäuser, Basel, 1992.

[37] L. Weis, Operator-valued Fourier multiplier theorems and maximal $L_{p}$-regularity, Math. Ann. 319 (2001), 735-758.

[38] H. Witvliet, Unconditional Schauder decompositions and multiplier theorems, Ph.D. thesis, Technische Univ. Delft, 2000.

[39] M. W. Wong, Wavelet Transforms and Localization Operators, Oper. Theory Adv. Appl. 136, Birkhäuser, Basel, 2002.

Institut für Mathematik

Universität Paderborn

Warburger Str. 100

33098 Paderborn, Germany

E-mail: cornelia.kaiser@uni-paderborn.de
Institut für Analysis Universität Karlsruhe Englerstraße 2

76128 Karlsruhe, Germany E-mail: lutz.weis@math.uni-karlsruhe.de

Received July 19, 2005

Revised version January 17, 2008 